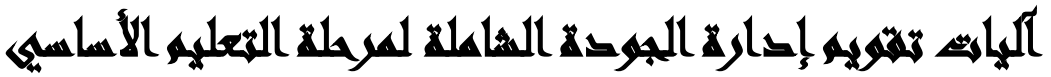

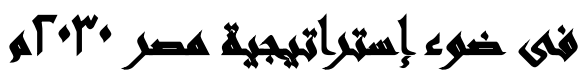

\author{
علا محمد خليفه(')- ممدوح عبد العزيز رفاعي(ץ)-- سوزان محمد المجدي(ז)

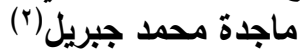

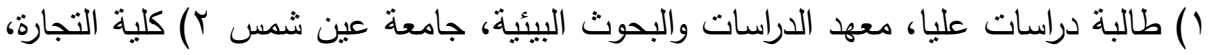

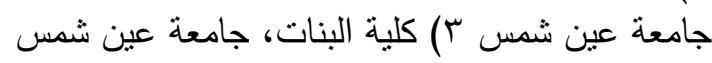

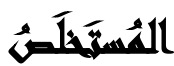

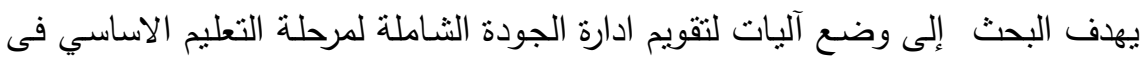

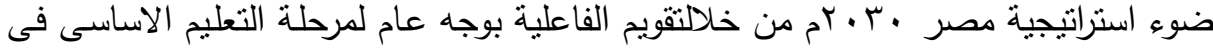

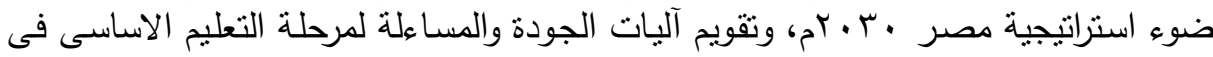

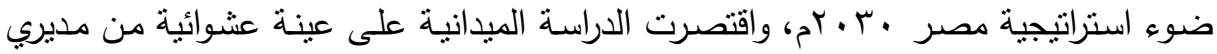

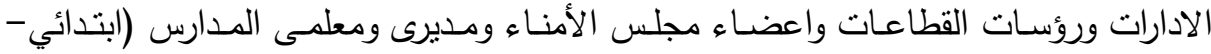

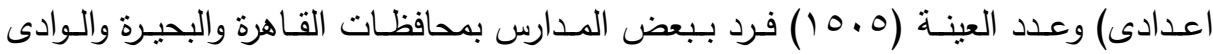

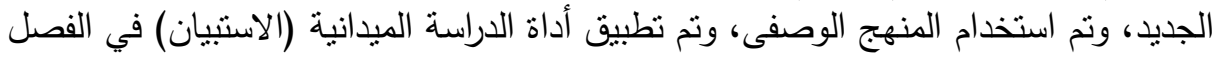

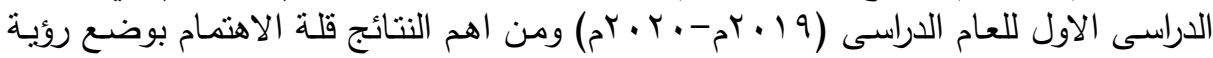

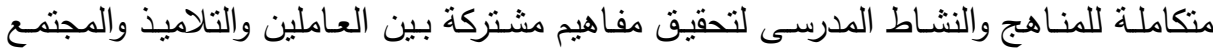

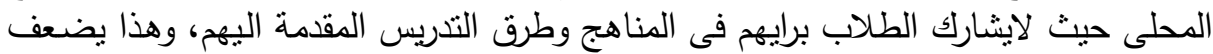

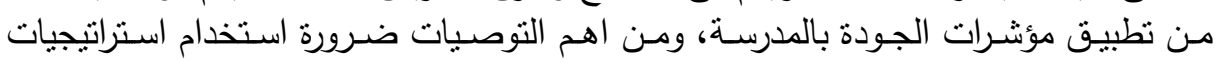

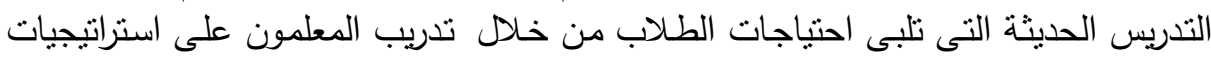
التدريس الحديثة، وتوفير التقتيات اللازمة لتطبيق استراتيجيات التنريس الحديثة. الكلمات المفتاحية: آليات تقويم إدارة الجودة الثناملة - التعليم الأساسي التئي 


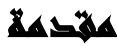

تتجه مصر إلى تطوير التعليم وتحديثه عن طريق إدارة تربوية تواكب منطلبات العصر ومواكبة التطورات التكنولوجية التي يشهدها العالم اليوم، والتي تؤكد على الجودة والقدرة التتافسية ودورها في العملية الإدارية ومدى مساهمة هذا الجانب في تحقيق أهداف المؤسسات

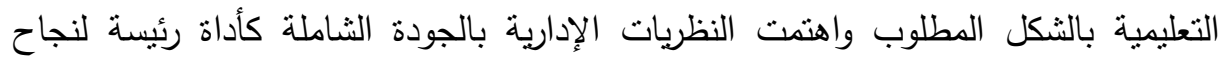

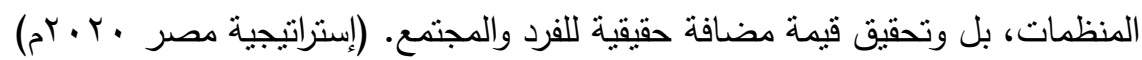
وفى ظل رؤية سيادة الرئيس عبد الفتاح السيسى لنطوير التعليم المصري والذي جعله على إلى

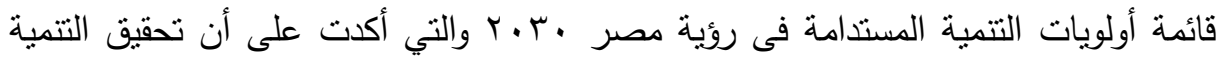
المنشودة في المجتمع يتوقف على وجود المنظمات القادرة على تحقيق أهدافها والوفاء

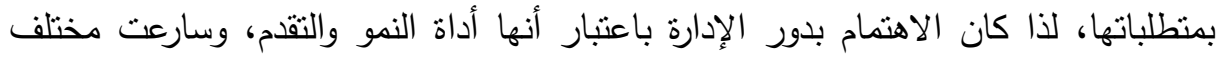

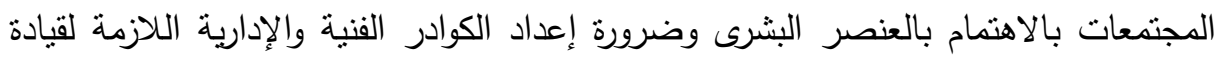
العمل بمنظمات المجتمع المختلفة باختلاف تخصصاتها وتبعيتها، واستشراف منظومة تتتاول الاتجاهات التربوبة الحديثة.

والتعليم جزء من عملية التتمية الثناملة مما يجعل المجتمعات تعمل بصورة منواصلة على مراجعة نظمها التعليمية، وإدخال تحسينات عليها، وعلى مناهجها الدراسية وتتبني العديد من الدول صيغة التعليم الأساسي ضمن آليات التحسين والإصلاح، والخروج بالتعليم من إطاره

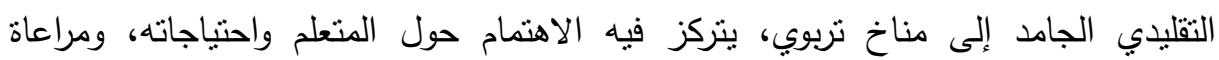

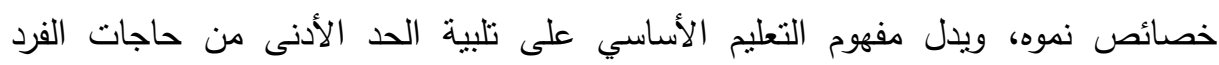

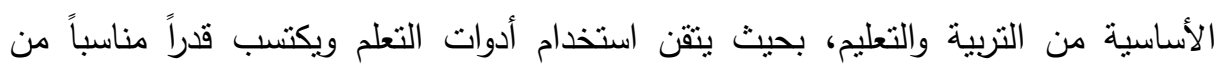

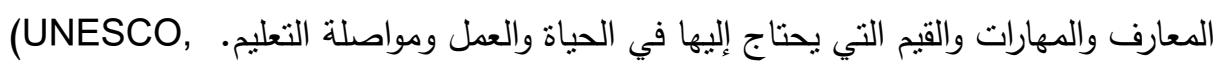




\section{And Illax}

ومن خلال عمل الباحثون فى مجال التربية والتعليم تواجه مرحلة التعليم الاساسى بجمهورية مصر العربية عدة مشكلات فى إيجاد آليات التقويم من اجل تحسين الكفاءة

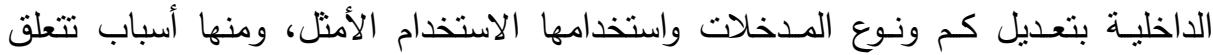
بالمؤسسات التعليمية نفسها والاعتماد علي طرق التدريس التقليدية، وغياب التتمية المهنية وانعدام الربط بين البرامج التدريبية والاحتياجات التتموية وضعف قدرة الإدارة المدرسية علي إحداث الإصـلاح المدرسي في جودة العملية التعليمية، وقلة التفاهم والتكامل في الأدوار الوظيفية الداخلية، وبالتالي يؤثر ذلك على القدرة المؤسسية التي تسعى إلى توفير أعلى

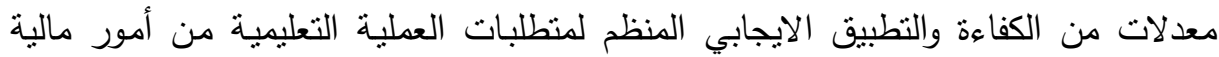
وفنية وتتظيمية وغيرها، أما إذا نقاعست الإدارة التعليمية عن أداء دورها في خدمة التعليم فإنها يصبح عبئا عليه وتقل بالتالي قدرة النظام التعليمي ويصيبه الضعف والقصيه التصور عن أداء دوره بالإضافة إلى ضعف كفاءته الداخلية، ويسعى الباحثون إلى إيجاد آليات تقويم فعالة لإدارة

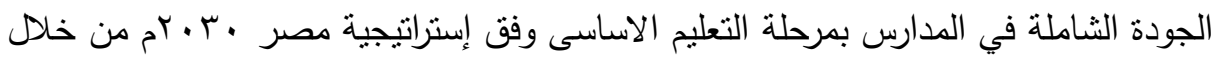
تقويم الفاعلية والقدرة المؤسسية. وقام الباحثون بدراسة استطلاعية وذلك بهدف استكمال الدراسات النظرية والتعرف على

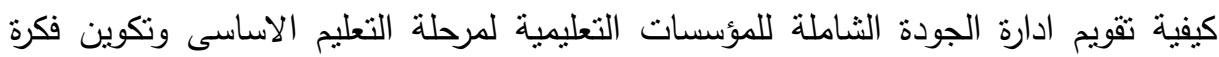

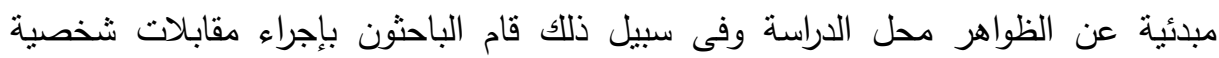
منظمة مع عينة من المعلمين في المدارس المختلفة وتم فى هذه المقابلة طرح بعض الأسئلة المتعلقة بموضوع البحث كالتالي: من عينه 
مجلة العلوم البيئية

معهد الدراسات والبحوث البيئية - جامعة عين شمس لئه

علا محمد خليفه وآخرون

جدول (1) : نسب الموافقة على أسئلة الدراسة الاسنطلاعية

\begin{tabular}{|c|c|c|c|}
\hline نسبة الموافقة & عدد الاشخاص & مجالات الاسئلة الموجهة للعينة & م \\
\hline ro & $1 \ldots$ & المدرسدة. التفاهم والتكاملـل في الأدوار الوظيفيـة الداخليـة للجودة فى & 1 \\
\hline$\varepsilon$. & $1 \ldots$ & 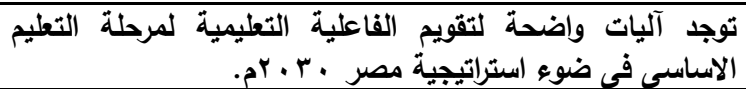 & r \\
\hline r. & $1 \ldots$ & 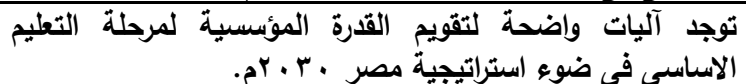 & $r$ \\
\hline
\end{tabular}

ومن الجدول السابق يتضح أن نسبة الموافقة منخفضة مما يشير إلى وجود قصور فى آليات ادارة الجودة الثناملة للمؤسسات التعليمية.

\section{أهمية المهمه}

تكمن أهمبة البحث فيما يلي:

ا-من المأمول أن بساعد البحث واضعى السياسات ومتخذى القرار في الإدارة التربوية على لهئ

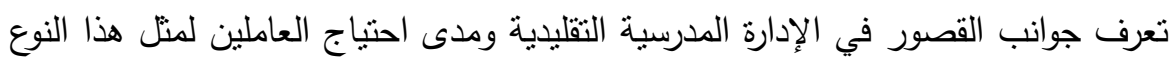
من الإدارة لكى يكونوا قادرين على أداء أدوارهم على أكمل وجله الإدأل

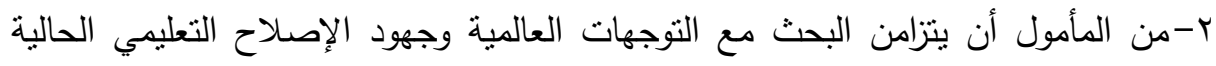

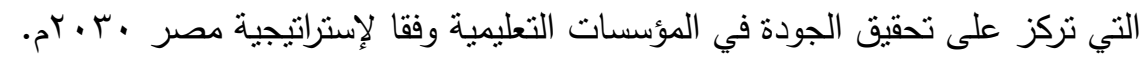

\section{أمساهن الهميه}

يهدف البحث إلى تقويم ادارة الجودة الثاملة لمرحلة التعليم الاساسي فى ضوء استراتيجية مصر •r • rم من خلال:

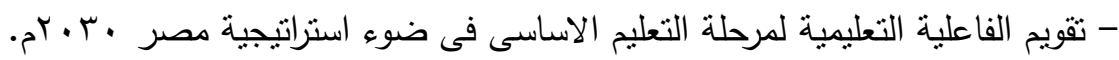

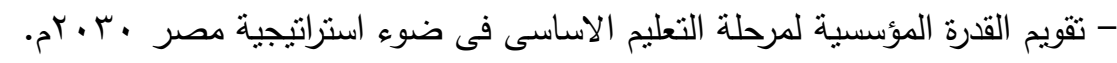




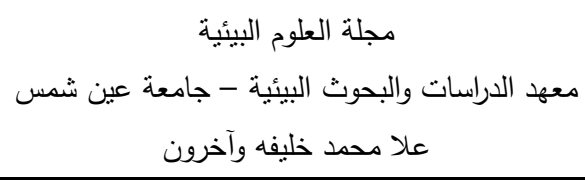

\section{هزونه المهنه}

- توجد علاقة ارتباطية بين تقويم الفاعلية التعليمية لمرحلة التعليم الاساسى وتحقيق متطلبات

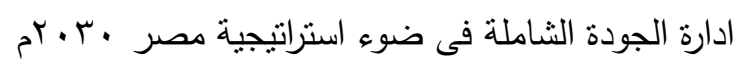

- توجد علاقة ارتباطية بين تقويم القدرة المؤسسية لمرحلة التعليم الاساسى وتحقيق منطلبات

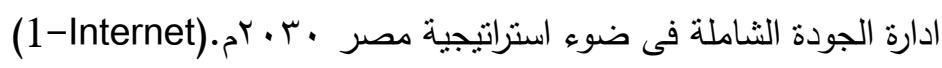

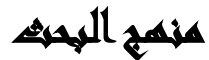

استخدم الباحثون المنهج الوصفي حيث كان ذلك أكثر ملائمة لطبيعة البحث.

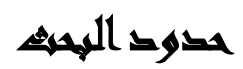

تحدد البحث بالحدود التالية:

الحدود الموضوعية: اقتصر البحث على مجالات ادارة الجودة الثاملة (تقويم الفاعلية بوجه

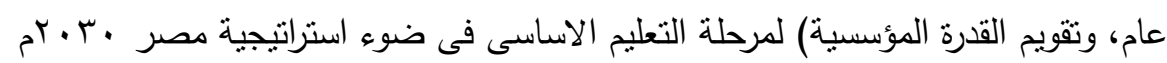

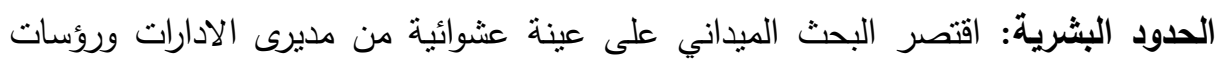
القطاعات واعضاء مجلس الامناء ومديرى ومعلمى المدارس (ابتدائى - اعدادى). الحدود المكانية: بعض المدارس بمحافظات القاهرة والبحيرة والوادى الجديد.

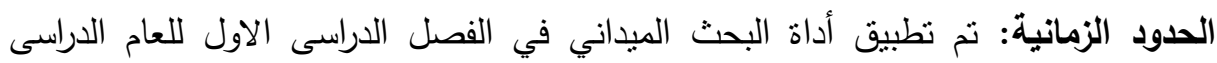

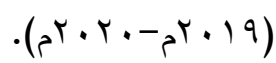
مجتمع البحث: يتكون مجتمع البحث من: 1- رؤساء قطاعات مديرية التربية والتعليم محافظات القاهرة - البحيرة - الوادي الجديد. r- أعضاء مجالس أمناء المدارس. r- م- مديروا المدارس. ع- - المعلمون في المدارس. 


$$
\begin{aligned}
& \text { مجلة العلوم البيئية } \\
& \text { معهد الدراسات والبحوث البيئية - جامعة عين شمس له } \\
& \text { علا محمد خليفه وآخرون }
\end{aligned}
$$

\section{همطلحاهي الهيه}

إدارة الجودة الثاملة:Thotal Quality Management

فلسفة إدارية حديثة تأخذ شكل نهج أو نظام إداري شامل قائم على أساس إحداث تغيرات إيجابية جذرية لكل شئ داخل المؤسسة بحيث تتمل هذه التغيرات الفكر والسلوك والقيم والمعتقدات التتظيمية والمفاهيم الإدارية ونمط القيادة الإدارية ونظم وإجراءات العمل المختلفة وذلك من أجل تحسين وتطوير كل مكونات المؤسسة للوصول إلى أعلى جودة في مخرجاتها.

التعليم الأساسى:Basic Education

هو المجموعة الكاملة للأنشطة التعليمية المندرجة في أطر مختلفة (التعليم النظامي والتعليم غير النظامي) والرامية الى تلبية احتياجات التعلم الاساسية، (القرائية والتعلم الثنفهي

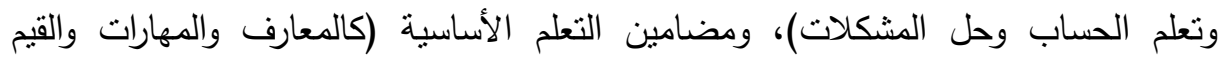

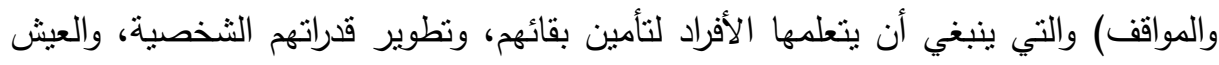

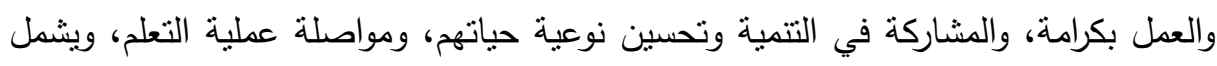

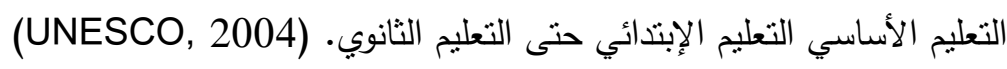

$$
\text { الاستراتيجية:-Strategy }
$$

مجموعة القرارات والنشاطات المختلفة المتعلقة باختيار الوسائل لتحقيق أهداف محددة

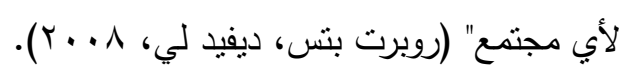

\section{التراسايت الماريها}

$$
\text { أولا: الاراسات العربية: }
$$

1-قامت سحر محمد أبو راضى عام 10 ب بدراسة هدفت الى الوقوف على الممارسات

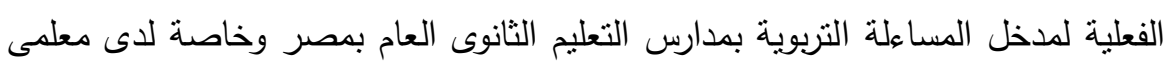
هذه المدارس، وطبقت الدراسة على عينة عشوائية من معلمى محافظة القليوبية بلغ عددهم 
( . (1) معلم، وتوصلت الدراسة لضعف اهتمام المدرسة بنشر ثقافة المساءلة بين أفرادها وتوضيح طبيعتها وأهدافها وأساليبها وآلياتها لجميع العاملين، وضعف وضئ وضوامح آلية المساءلة

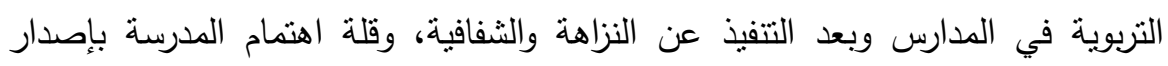
قوانين جديدة تضمن حقوق العاملين، وضعف مشاركة العاملين في تحديد معايير الأداء وتدنى ثقافة الجودة لديهم.

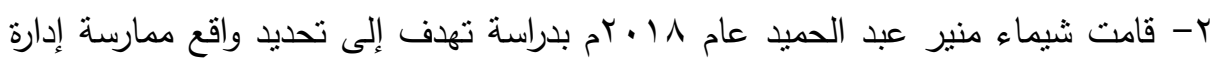
المدرسة الثانوية للإدارة بالحب داخل المدرسة والتحديات التي تواجه نطوير القدرة المؤسية بالمدرسة الثنانوية العامة، استخدمت المنهج الوصفى، والاستبيان كاداة لجمع البيانات، عينة الدراسة (ب. ب) فرد من معلمي ومديرى المدارس الثانوية، ومن أهم نتائج الدراسة توفر الإدارة بالحب مناخ مؤسسى محب للعاملين يزيد من ولائهم وانتماؤهم للمدرسة المانة ورفع كفائته الإنتاجية وتهنم برأس المال البشرى باعتباره الثروة الحقيقية وأساس عملية باسنية التطوير المؤسسي وأساس الإبداع والابتكار والمشاركة الفعلية فى الخدمات الاجتماعية باعناعية وباعنبارها مدخلا للنطوير والتحسين المستمر • ثانيا: الدراسات الأجنبية:

ا-قامت تينا هويلي Tina Hoyle عام ^ .. بام بدراسة استهدفت توضيح الثروط اللازمة لبناء قدرة الددارس والتي تسهم في استمرار بقائها، وقد طبقت الدراسة على مدرسة بيبلو كووردو في الولايات المتحدة الأمريكية؛ وذللك لمعرفة جهودها والتطوير والتحسين المستمرين في تطوير الصحة في المجتمع المدرسي، وتوصلت إلى مجموعة من النتائج

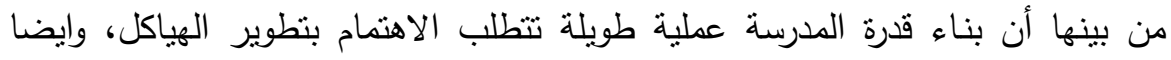

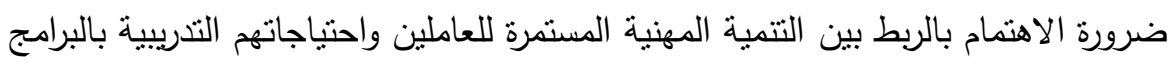

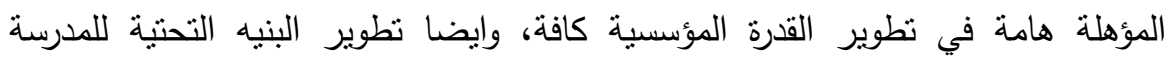
باستمرار يزيد من قدرتها المؤسسية. 
r-قامت ليندا ريدى وآخرون Linda Reddy, etc عام 0 • ب بم بدراسة استهدفت التعرف على الإطار الفكرى للقدرة المؤسسية والتسويق الإستراتيجى للمدرسة الثانوية العامة،

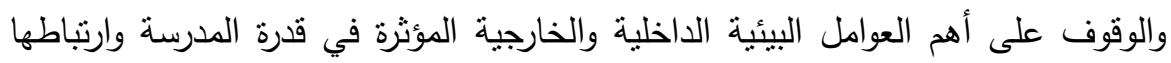

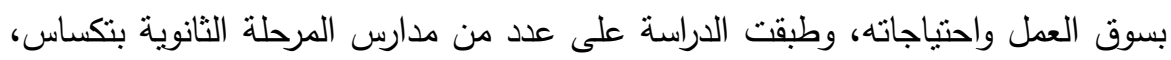
واستخدمت الدراسة أسلوب التحليل البيئى، وتوصلت الدراسة إلى إستراتيجية مقترحة لتطوير القدرة المؤسسية بالمدرسة الثانوية لتحسين تسويقها الإستراتيجي، وأكدت على تأثير الاهتمام بجودة الخدمات المقدمة على كسب عملاء جدد وتحسين التسويق الإستراتيجي.

\section{الإلطار اللنظيه}

تتاول البحث في جانبه النظري إدارة الجودة الثاملة لمرحلة التعليم الأساسي في ضوء

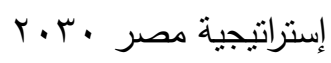

أما إذا أردنا التحدث عن الجودة بصورة عامة ومحاولة إيجاد مفهوم يعبر عن هذا

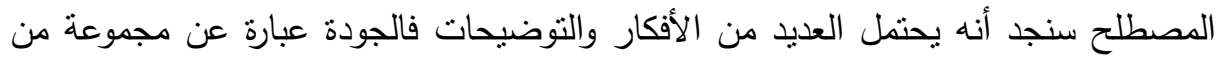

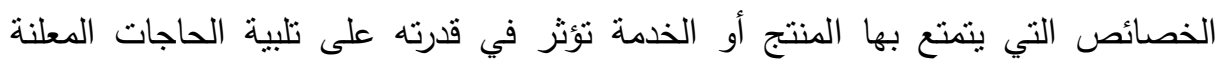
والضمنية من استخدامه وهذه الخصائص تتغير حسب الوقت وبالتالي لابد من المراجعة المستمرة لمنطلبات الجودة وهذه المتطلبات عادة ما ينت نرجمتها إلى خصائص ومعايير فهناك من قال أن الجودة هي :الإتقان في العمل. أو الأداء الصحيح وبطريقة صحيحة منذ المرة لرهة الأولى.او" عمل الأشياء بطريقة صحيحة من المرة الأولى، وبطريقة أفضل في المرة التالية."

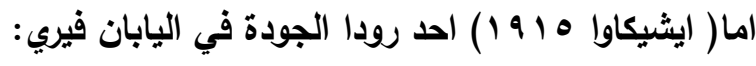
- إن الجودة قد يتسع مداها لتشمل جودة العمل والخدمة والمعلومات والتشغيل والنظام وجودة عمل الموظفين، وجودة الشركة وغير ذلك، مما يجعل مناظرة الجودة وأبعادها

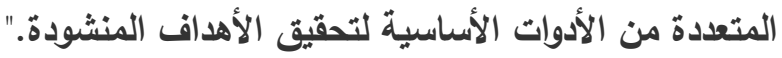


واستخدت ISO 9001/9002/9003 المواصفات الدولية بإصدارها القديم مصطلح

نظم تأكيد الجودة (Quality Assurance Systems)، فيما استخدمت بsO

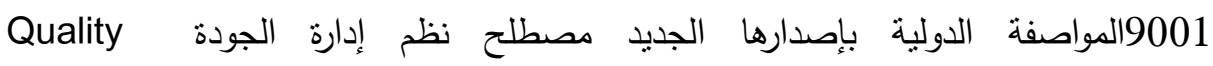
Management System وهو مؤشر على التطور في استخدام المفهوم، إذ أن إدارة الجودة

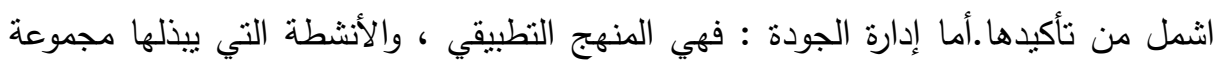

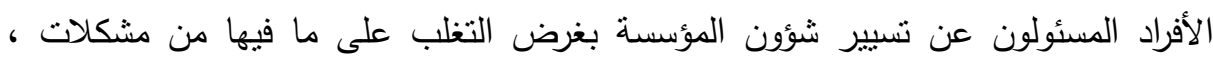
والمساهمة بشكل مباشر في تحقيق النتائج المرجوة وتحقيق حاجات وتوقعات العميل ؛ وبالتالي فهي عملية مستمرة لتحسين المنتجات والمحافظة عليها (Frederick, 1996) ، إن منظمات الأعمال بمختلف إثكالها وأحجامها في ظل البيئة التتافسية الثديدة التي أصبحت تعيش فيها، وبفضل ما أفرزته ثورة الاتصالات والمعلومات والتطورات التقنية المستمرة، وظهور العديد من فئن

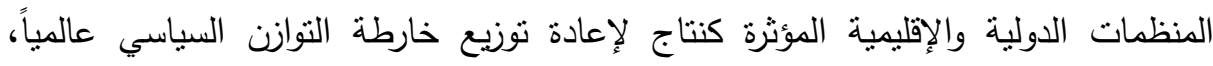

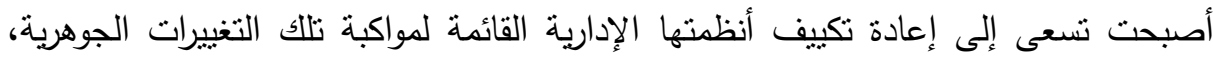
وللمحافظة على مكانتها في السوق بعد أن أصبحت الجودة والارتقاء بها أحد أهم المحددات

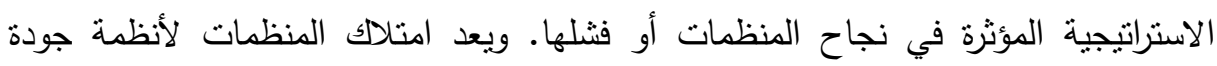
فاعلة تؤئر على جودة منتجاتها وخدماتها، وتتفاعل ايجابياً بصورة تكاملية مرنة مع الممارسات

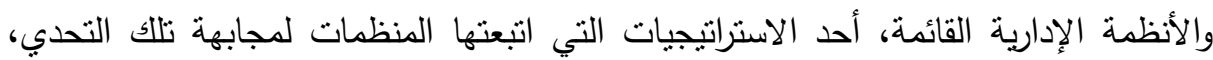

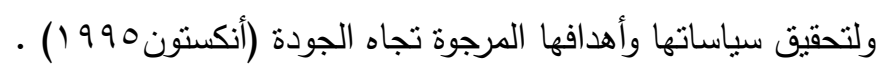
اذا لا بد من إيجاد نقافة جديدة داخل المنظمة، ثقافة تركز بقوةعلى إثباع رغبات العملاء و تهنم بذلك؛ إنها ثقافة إثباع رغبات العملاء وذللك عن طريق التحسين والتطوير المستمرفي عمليات وأنشطة المنظمة، حتى يمكن تحقيق وفر في التكاليف وسرعة أعلى في

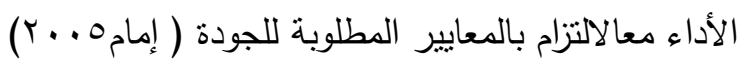


كما يعتبر الأفراد العاملون في المنظمة هم المحور الرئيسي الذي تقوم عليه عملية|إتقان

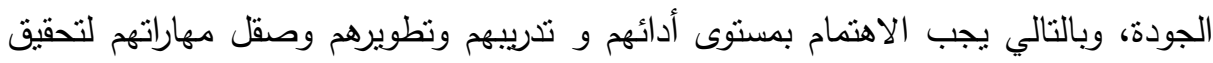
المستوى المطلوب من الجودة .

ثم إن تضافر جهود الأفراد تظهر في أحسن صورها من خلاد بناء فرق العمل و تشجيع

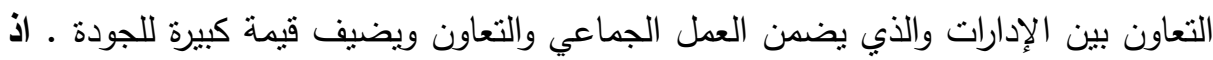
يحتاج تحقيق مستويات الجودة إلى الإبداع والابتكار وإلى إطلاق أكبر عدد ممكن من الأفكار الجديدة والمفيدة لتحسين الجودة .

كما انه لا بد من وجود رؤية إستراتيجية للمنظمة ككل حول كيفية تحقيق الجودة مع ربط هذه الإستراتيجية بكافة أنشطة المنظمة ـ ثم انه لابد من تعليم الإدارة والعاملين كيفية تحديد

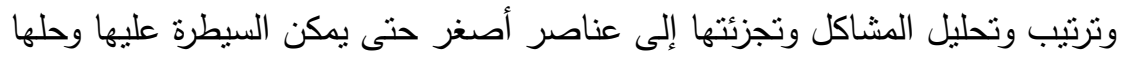
وتُعرف إدارة الجودة الثاملة TQM بأنها مدخل لإدارة المنظمة، برتكز على على الجودة،

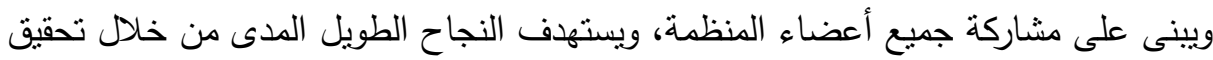
رضاء العميل وتحقيق منافع لجميع أعضاء المنظمة والمجتمع.( النجار 19919 1 ) ووفقا ل (Dale\& Plunkett, 1995) فان مراحل تظور إدارة الجودة الثاملة

Inspection تتضمن: الفحص

وتتضمن أنشطة الفحص: الفرز، التصنيف، اكتشاف العيوب، الإجراءات التصحيحي ومراقبة الجودة Quality Control وهي الأنشطة والأساليب التشغيلية التي تُشتخدم لإتمام منطلبات الجودة، وتتضمن أنشطة مراقبة الجودة: اختبار المنتج، بيانات أداء العملية،

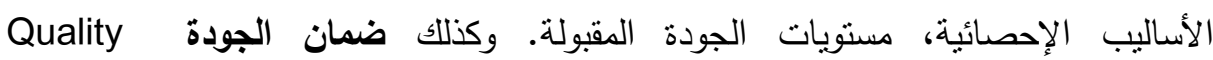
Assurance

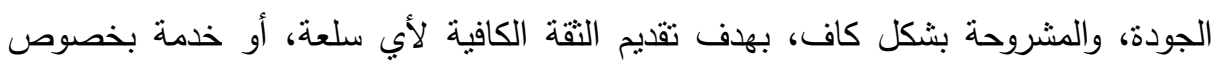
كونها تلبي متطلبات الجودة، وكذلك إدارة الجودة الثاملةTQM ويشمل ضمان الجودة 
الأنشطة الآتية: تخطيط الجودة، نطوير نظم الجودة، إعداد دليل الجودة، المراقبة الإحصائية

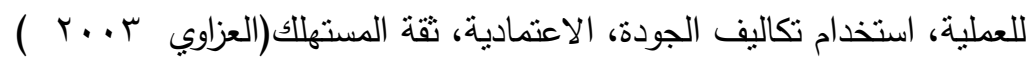
وتتضمن إدارة الجودة الثاملة: التحسين المستمر، التركيز على العميل الداخلي التهاليه والخارجي والمجتمع، المشاركة، علاقات التعاون، حلقات الجودة، فرق التحسين عبر الإدارات، نشر وظيفة الجودة، الاتصالات المفتوحة، التميز الدائم، حل المشكلات من مصدرها، رضاء التهاء العملاء المستمر، أداء العمل بشكل صحيح من أول مرة وفي كل مرة، وفي الوقت المنات التاسب.

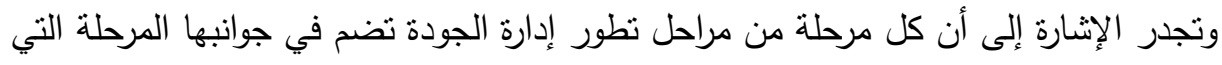
تسبقها، فمرحلة مراقبة الجودة تتضمن الفحص، ومرحلة ضمان الجودة تتضمن مراقية مراقبة

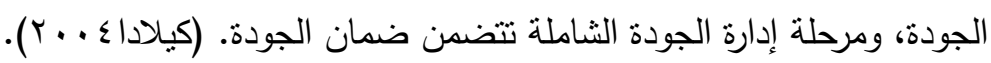
ولإن التحول إلى إدارة الجودة الثناملة يتطلب إجراء تغييرات في الثنافة التنظيمية

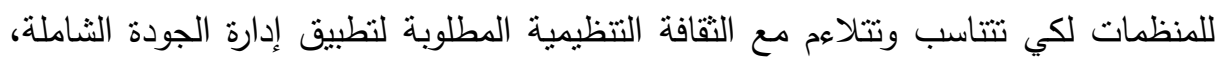
فان إجراء مثل هذه التغييرات يتطلب مشاركة جميع العاملين في الأمور المتعلقة بالجودة وبالمنظمة ككل ، وتركز ثقافة المنظمات التي تتوجه بإدارة الجودة الثاملة على التحسين المستمر في العمليات لتحقيق رضاء العملاء الداخليين والخارجيين، وعلى الأهداف طويلة

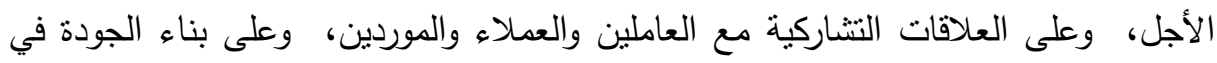
مرحلة التصميم، وعلى الاستثمار في أنشطة الوقاية بهدف تخفيض تكاليف الفشل الداخلي والخارجي، كما تركز على تمكين العاملين وتتكيل فرق عمل، وعلى الصيانة الإنتاجية

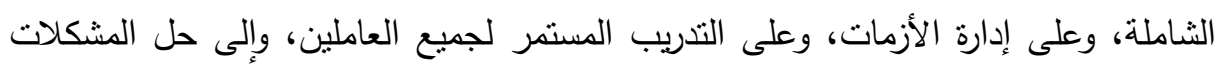
من مصدررا (, Juran \&Gryna1998). وهناك ثلاثة طرق لتعريف إدارة الجودة الشاملة: يتمركز تعريف الطريقة الأولي حول منطلبات واحتياجات وتوقعات العميل فعرفها كول على أنها " نظام يشنتل على مجموعة من الفلسفات المتكاملة والأدوات الإحصائية والعمليات الإدارية المستخدمة لتحقيق الأهداف ورفع مستوى رضا العميل والموظف على على حد سواء".

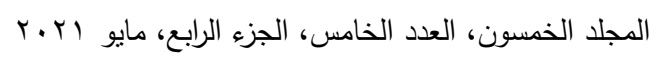


كما أنها " انتراك والتزام الإدارة والموظف في ترشيد العمل عن طريق توفير ما يتوقعه العميل وما يفوق توقعاته".

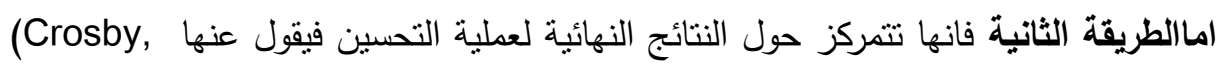
(1996" أنها الطريقة المنهجية المنظمة لضمان سير النشاطات التي خطط لها مسبقا وهي

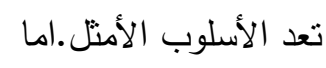

الطريقة الثالثة لتعريف إدارة الجودة الثاملة فتتمحور حول فكرة استخدام الوسائل العلمبة والأدوات الإحصائية المتاحة لتطبيق مفهوم الجودة الثاملة.حيث قال عنها محفوظ جوده نقلا

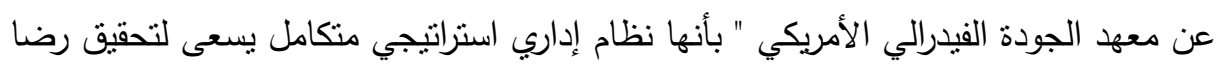
العميل وإن تطبيق هذه الفلسفة الإدارية يحتم مشاركة جميع المديرين والموظفين ويقوم

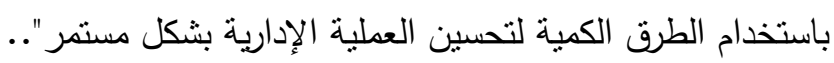
وتجنب حدوث المشكلات من خلال التشجيع على السلوكيات الجيدة والاستخدام الأمنل

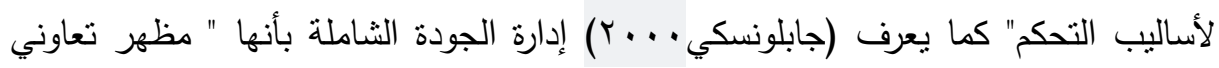

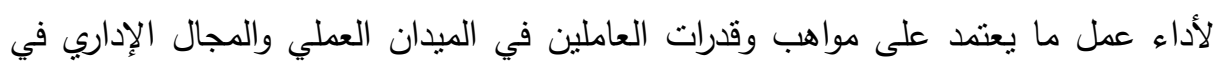
سبيل توفير تحسين جودة مستمر وإعداد فرق عمل ذات كفاءة إنتاجية عالية". ويدور الاستطلاع حول محوري البحث وهما:

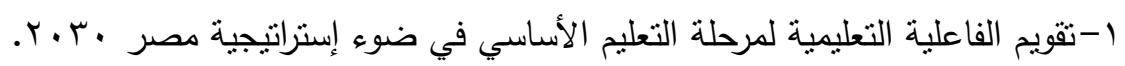

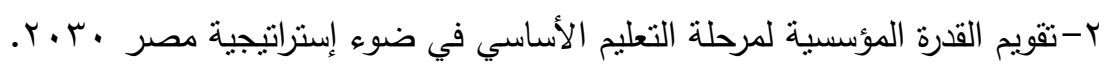

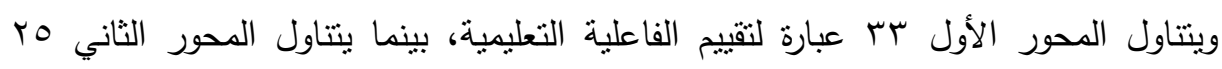

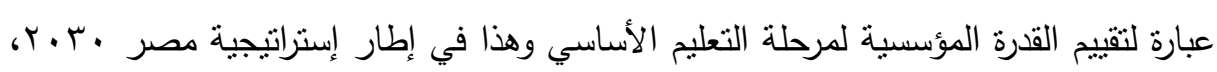
وقد تم عرض الاستمارة على خبراء في التعليم والإدارة المدرسية للوصول إلى الطى استمارة

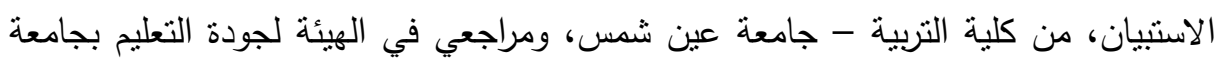




\section{إجبراعايت الهيهA}

منهج البحث: استخدم الباحثون المنهج الوصفي (الدراسات المسحية) حيث كان ذلك أكثر مالائمة لطبيعة البحث. مجتمع البحث: يتكون مجتمع البحث من (رؤساء القطاعات بمديرية التربية والتعليم

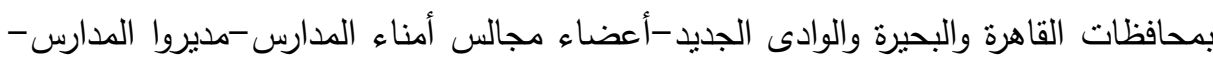
المعلمون فى المدارس)

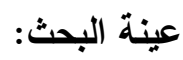

جدول (ץ): توصيف عينة البحث الأساسية والاستطلاعية

\begin{tabular}{|c|c|c|c|}
\hline الاستطلاعية & الأساسية & عينة البحث & r \\
\hline 1 . & Yos & رالبحيرة والوادى القطاعات بمديرية التربية والتعليم بمحافظات القاهرة & 1 \\
\hline$\wedge$ & 187 & أعضاء مجالس أمناء المدارس & r \\
\hline$\checkmark$ & V) & مديروا المدارس & r \\
\hline ro & $90 \leqslant$ & المعلمون في المدارس & $\varepsilon$ \\
\hline 0. & $1 \leqslant 00$ & 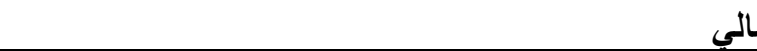 & الإم \\
\hline
\end{tabular}

أدوات جمع البيانات:

- إستمارة الإستبيان: ومن خلال الخطوات السابقه تمكن الباحثون من تحديد محاور وأبعاد

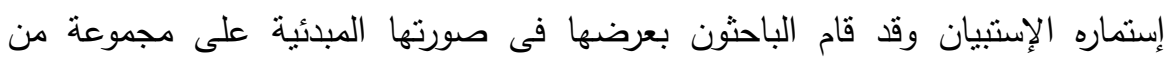

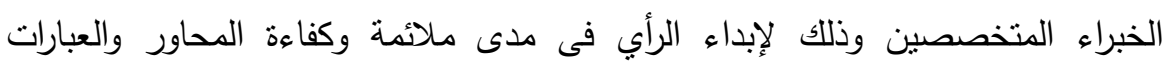
المقترحة لتحقيق أهداف البحث. 
رابعاً: المعاملات العلميه لإستمارة الإستبيان:

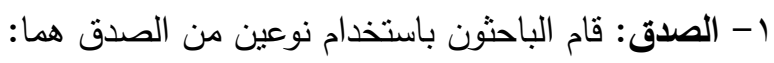

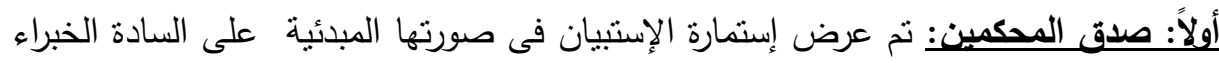
لابداء الرأى فى ملائمة وكفاية المحاور والعبارات المقترحه، والتأكد من سلامة الصياغة الصنيان

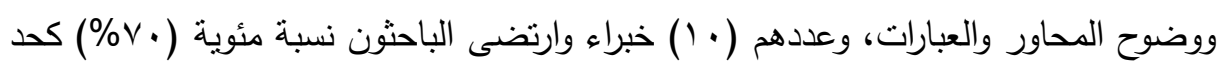

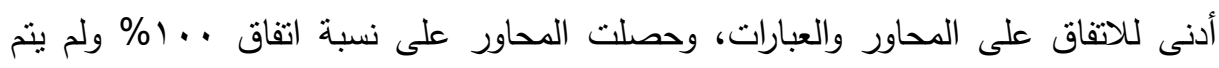
التعديل او الحذف، وتم عرض العبارات تحت كل محور وفيما يلى عرض نسبة اتفاق الخبراء على العبارات.

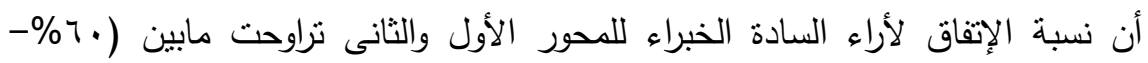

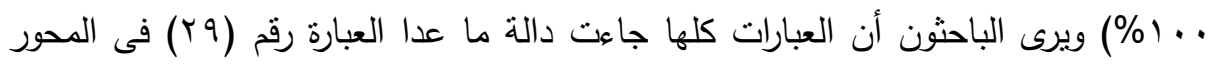

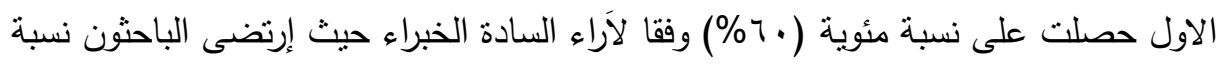

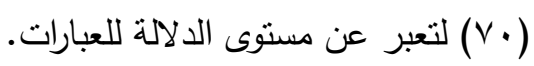
ثانيا: صدق الاتساق الاخلي:تم التحقق من صدق الاستبيان عن طريق استخدام صدق لاتقات

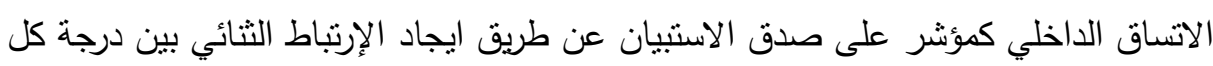

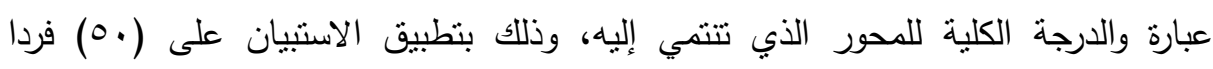
والجداول الآتية توضح هذه النتائج: 
مجلة العلوم البيئية

معهد الدراسات والبحوث البيئية - جامعة عين شمس لئن

علا محمد خليفه وآخرون

جدول (ץ): نسبة الاتفاق لأراء السادة الخبراء لعبارات المحاور

\begin{tabular}{|c|c|c|c|c|c|c|c|c|c|}
\hline \multicolumn{4}{|c|}{ المحور الثانح } & \multicolumn{6}{|c|}{ المحور الاول } \\
\hline م.الارتباط & P & م.الارتباط & 5 & م.الارتباط & 5 & م.الارتباط & 5 & م.الارتباط & 5 \\
\hline · & iv & $\bar{e} \cdot, Y Y \cdot$ & 1 & - TVO & Tו & $\bar{e} \cdot, \Sigma \wedge \wedge$ & IV & $\cdot, V \leq Y$ & 1 \\
\hline$\cdot, T \leqslant V$ & 11 & . ह ₹qr & $r$ & $\cdot, \varepsilon \vee$. & r & •, हq & 11 & $\cdot, 7 Y 1$ & $r$ \\
\hline$\cdot, \leqslant Y 0$ & 19 & $\cdot, \leqslant \vee q$ & 7 & $\cdot, \leqslant 97$ & אי & •, $\_0 r$ & 19 &., $0 Y$. & 7 \\
\hline$\cdot \varepsilon r V$ & $r$. & $\cdot, \leqslant 00$ & $\varepsilon$ & $\cdot, \wedge r V$ & $r \varepsilon$ &., 071 & $r$. & $\cdot,\{91$ & $\varepsilon$ \\
\hline$\cdot, \leqslant 01$ & YI &., 019 & 0 & & & • & rI &.,$O Y V$ & 0 \\
\hline$\cdot, \leqslant \vee 0$ & Tr &., $07 \mathrm{~V}$ & V & & & $\cdot, 7 \leq 1$ & Tr & $\cdot, \wedge 7 ו$ & V \\
\hline$\cdot, 7 \wedge T$ & Tr & $\cdot, 0 \wedge 9$ & $\Lambda$ & & &., $0 . r$ & rT & $\cdot, \wedge \wedge \varepsilon$ & $\Lambda$ \\
\hline., $07 \mathrm{~V}$ & T纟 & $\cdot, \leqslant 0$. & 9 & & & $\cdot, \wedge \vee\urcorner$ & $r \xi$ & $\cdot, \wedge \vee$. & 9 \\
\hline$\cdot, \Sigma \vee 1$ & To & $\cdot, \varepsilon \Gamma \wedge$ & 1. & & & $\cdot, \wedge Y V$ & ro &., $07 \mathrm{~V}$ & 1. \\
\hline & & $\cdot, \vee \vee \cdot$ & 11 & & & $\cdot, 7 \vee \wedge$ & rY &., 790 & 11 \\
\hline & &., 0.1 & IT & & &., 110 & TV & $\cdot, 7 \wedge 9$ & 14 \\
\hline & & $\cdot,\{7\}$ & $\pi$ & & &., $09 \pi$ & rA &., 77. & Tr \\
\hline & & $\cdot, \varepsilon O r$ & $1 \varepsilon$ & & & $\cdot, T Y I$ & rq & $\cdot, 7 \wedge 7$ & $1 \varepsilon$ \\
\hline & & $\cdot, r \wedge 9$ & 10 & & & $\cdot, 7 \leqslant \varepsilon$ & $r$. & $\cdot, v \cdot 9$ & 10 \\
\hline & & •,АYт & 17 & & & $\cdot, V \backslash V$ & M & $\cdot, 0.1$ & 17 \\
\hline
\end{tabular}

يتضح من جدول (r) ان قيم معاملات الارتباط لعبارات المحور الاول جاءت دالة

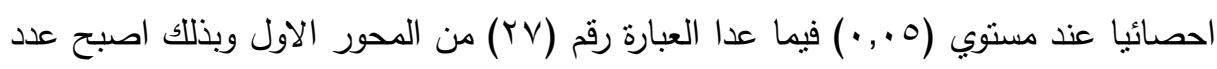

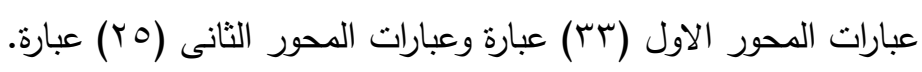
الثبات: قام الباحثون باستخدام معامل (الفاكرونباخ)

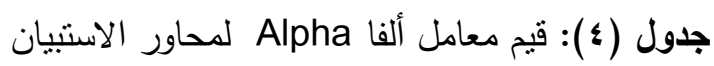

\begin{tabular}{|c|c|c|}
\hline قيمة معامل ألفا & مسمى المحور & المحور \\
\hline$\cdot, \wedge \vee r$ & تقويم الفاعلية التعليمية لمرحلة التعليم الاساسى فى ضوء استراتيجية & الأول \\
\hline .,人т0 & ثقويم القدرة المؤسسية لمرحلة التعليم الاساسى فى ضوء استراتيجية . مصر. & الثاني \\
\hline
\end{tabular}

المجلد الخمسون، العدد الخامس، الجزء الرابع، مايو الr.r.

الترقيم الدولي 0826-1110 110 


$$
\begin{aligned}
& \text { مجلة العلوم البيئية } \\
& \text { معهد الدراسات والبحوث البيئية - جامعة عين شمس به } \\
& \text { علا محمد خليفه وآخرون }
\end{aligned}
$$

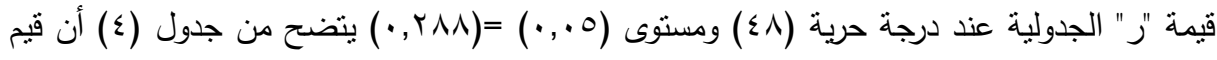

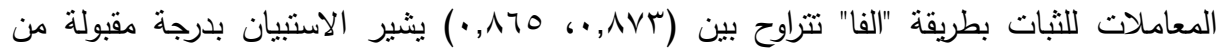

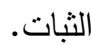

الإجراءات المنهجية للبحث: 1-نوع الدراسة: تعتبر الدراسة من الدراسات الوصفية لأنها أنسب أنواع الدراسات الملائمة

$$
\text { لطبيعة موضوع البحث. }
$$

ץ-منهج البحث: المستخدم هو منهج المسح الاجتماعي بالعينة من خلال استخدام عينة من

$$
\text { مجتمع البحث. مكان. }
$$

أ- - مكان الاراسة: تم عمل البحث في محافظات القاهرة -البحيرة- الوادي الجديد

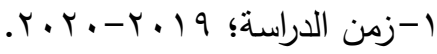
عرض ومناقثة وتفسير نتائج المحور الأول:

يتعلق المحور الأول بتقويم الفاعلية التعليمية بالتعليم الأساسي في ضونية ضوثي إستراتيجية مصر .r.r. 
مجلة العلوم البيئية

معهد الدراسات والبحوث البيئية - جامعة عين شمس البية

علا محمد خليفه وآخرون

جدول (0): التكرارات والاهمية النسبية وكاب لعبارات المحور الاول (ن=00؛ ( )

\begin{tabular}{|c|c|c|c|c|c|c|c|c|c|c|c|c|c|}
\hline \multirow[t]{2}{*}{ SL } & \multirow{2}{*}{ الالامية } & \multirow{2}{*}{ اللرجات } & \multicolumn{2}{|c|}{ غير موافق } & \multicolumn{2}{|c|}{ غير موافق } & \multicolumn{2}{|c|}{ أوافق الى حـ } & \multicolumn{2}{|c|}{ اوفق } & \multicolumn{2}{|c|}{ أوافق بشدة } & \multirow[t]{2}{*}{ العبــارة } \\
\hline & & & $\%$ & 5 & $\%$ & s & $\%$ & 5 & $\%$ & s & $\%$ & s & \\
\hline or, & $v_{0, r}$ & $0 \leqslant \vee q$ & $\varepsilon, \cdot$ & $0 \wedge$ & $v, 1$ & $1 . r$ & $r \cdot r$ & « 1 & ro, $T$ & rVr & r & $\varepsilon \wedge$. & 1 \\
\hline 079,9 & Vo, v & 00.7 & $r, r$ & $\leqslant \wedge$ & $7, \varepsilon$ & $q \pi$ & ru,. & $\{01$ & $r V, r$ & Tат & $r, 1$ & $\leqslant 7 V$ & $r$ \\
\hline 7.A, & V7, & oors & $r, V$ & $\varepsilon$. & 0,1 & 10 & $r 1,0$ & $\varepsilon 09$ & $r \wedge, \cdot$ & $\varepsilon \cdot 1$ & $\Gamma, \Lambda$ & $\sum \pi$ & $r$ \\
\hline or.,. & $V \varepsilon, r$ & $0 \leqslant \cdot V$ & $r, q$ & ov & $\mathrm{V}, \cdot$ & $1 . r$ & $r \cdot, \varepsilon$ & $\varepsilon \leqslant Y$ & $r \cdot, q$ & 纟o. & $r V, \Lambda$ & $\varepsilon \cdot \varepsilon$ & $\varepsilon$ \\
\hline or4,V & $v_{0,}$. & $0 \leqslant 0 V$ & $r, \tau$ & or & $7, V$ & 91 & $r \cdot, v$ & $\leq \leqslant 7$ & $r \wedge, q$ & $\varepsilon r$. & $r \cdot, 1$ & $\varepsilon r \Lambda$ & 0 \\
\hline or $\Lambda, \varepsilon$ & $V \leqslant, \varepsilon$ & $0 \leqslant 11$ & $r, v$ & $0 \leqslant$ & 7,1 & 99 & $r \cdot, T$ & $\leq \leqslant 0$ & $r,, v$ & $\leq 71$ & $r V, r$ & $r 97$ & 7 \\
\hline or, r & $V \varepsilon, \varepsilon$ & $0 \leqslant 1 Y$ & $r, \Lambda$ & 00 & 7,9 & $1 \ldots$ & $r \cdot, 0$ & $\varepsilon \leqslant \varepsilon$ & $r, r$ & $\{00$ & $r V, T$ & $\varepsilon+1$ & $\mathrm{v}$ \\
\hline or4,9 & $v_{0, .}$ & $0 \leqslant 09$ & $r, 7$ & or & $7, \mathrm{~V}$ & 91 & $r \cdot, v$ & $\varepsilon \leqslant 7$ & $r \wedge, Y$ & $\sum 11$ & $r \cdot, r$ & $\leq \varepsilon$. & $\wedge$ \\
\hline $0 . v, \varepsilon$ & $V \varepsilon, \Lambda$ & $0 \leqslant \leqslant 0$ & $\varepsilon, 1$ & 7. & $V, r$ & 1.0 & $r \cdot, r$ & $\varepsilon r q$ & $r V, r$ & rav & $r, r$ & $\leqslant 0 \leqslant$ & 9 \\
\hline$\varepsilon v \cdot, 1$ & $V \varepsilon, Y$ & or 99 & $\varepsilon, 7$ & TV & $v, v$ & 111 & $r q, r$ & $\varepsilon r r$ & $r \wedge, \cdot$ & $\varepsilon \cdot 1$ & $r \cdot, \cdot$ & \& & 1. \\
\hline orr,. & $V 0, r$ & $0 \leqslant 71$ & $r, \Lambda$ & 00 & 7,9 & $1 \ldots$ & $r \cdot, 0$ & $\varepsilon \leqslant \varepsilon$ & $r V, \varepsilon$ & $r 99$ & $r, \varepsilon$ & 纟OV & 11 \\
\hline$\leq V_{0}, \varepsilon$ & $V r, T$ & oros & $\varepsilon, 7$ & TV & $V, V$ & 114 & $r q, r$ & $\varepsilon r r$ & $r 1,1$ & sor & $r 7,9$ & r91 & ir \\
\hline$\leqslant \vee \wedge, 0$ & $V r, \cdot$ & & $\varepsilon, \wedge$ & $v$. & $v, q$ & 110 & $r 9,0$ & $\varepsilon r q$ & rr, q & $\sum \vee q$ & $r \leqslant, q$ & T4 & $1 \pi$ \\
\hline ory & $V \varepsilon, V$ & $0 \leqslant T 4$ & $r, \Lambda$ & 00 & 7,9 & $1 \ldots$ & $r \cdot, 0$ & $\varepsilon \leq \varepsilon$ & $r q, 7$ & $\varepsilon r 1$ & $r q, r$ & Ero & $1 \leq$ \\
\hline 10 ג & vo,. & $0 \leqslant 01$ & $r, \tau$ & or & $T, V$ & 91 & $r \cdot, v$ & $\leqslant \leq 7$ & $r \wedge, \Lambda$ & $\leqslant 19$ & $r \cdot r$ & $\varepsilon r q$ & 10 \\
\hline orr, 9 & $V \varepsilon,$. & סTAT & $\varepsilon_{,}$. & 01 & $\mathrm{v}, \mathrm{l}$ & $1 . r$ & $r \cdot, r$ & «s & $r, Y$ & $\leqslant 79$ & $Y_{\uparrow, \xi}$ & $\Gamma_{\Lambda} \Sigma \varepsilon$ & 17 \\
\hline$\varepsilon \wedge \varepsilon, \vee$ & $V r, V$ & איז4 & $\varepsilon, 0$ & 70 & $v, \tau$ & 11. & $r q, \wedge$ & $\varepsilon r \leqslant$ & $r, r$ & $\leq 0 \leq$ & $r q, q$ & rar & IV \\
\hline or $৭, v$ & $V \leqslant, 7$ & $0 \leqslant Y_{0}$ & $r, \tau$ & or & $7, \mathrm{~V}$ & 91 & $r \cdot\rangle$, & $\leq \leqslant 7$ & $r, 1$ & sor & $r v, q$ & $\varepsilon .7$ & 11 \\
\hline or 9,0 & $V \leqslant, 0$ & $0 \leqslant 1 V$ & $r, \wedge$ & 00 & 7,9 & $1 \ldots$ & $r \cdot, 0$ & $\varepsilon \leqslant \varepsilon$ & $r \cdot, q$ & so. & $r v, q$ & $\varepsilon .7$ & 19 \\
\hline 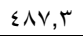 & $V \leqslant, 7$ & $0 \leqslant Y \leqslant$ & $\varepsilon, \varepsilon$ & $T \varepsilon$ & $\mathrm{v}, \mathrm{O}$ & 1.9 & $r q, q$ & $\leqslant r_{0}$ & $Y V, \varepsilon$ & ra1 & $r \cdot, q$ & $\leqslant \leqslant 9$ & r. \\
\hline$\varepsilon Y \leqslant, T$ & $V K, Y$ & orrs & $0, r$ & VV & $\Lambda, \varepsilon$ & MK & $r q,$. & 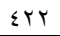 & $r q, 0$ & $\varepsilon r q$ & $r v, \Lambda$ & $\varepsilon .0$ & r) \\
\hline OrY,Y & $V \leqslant, A$ & $0 \leqslant \leqslant 1$ & $r, \Lambda$ & 00 & 7,9 & $1 \ldots$ & $r \cdot, 0$ & $\varepsilon \leqslant \varepsilon$ & $r q, r$ & EYT & $r q, 7$ & $\varepsilon r$. & rr \\
\hline$\leq 11, \varepsilon$ & $V r, 1$ & orlo & 0,0 & $\wedge$. & $\Lambda, 7$ & Iro & $r \Lambda, \Lambda$ & $\{19$ & $r q, r$ & $\varepsilon r V$ & $r V, \Lambda$ & $\varepsilon \cdot \varepsilon$ & r \\
\hline$\varepsilon \cdot r, \Lambda$ & $V r, r$ & Orrs & 0,7 & $\Lambda r$ & $\Lambda, \mathrm{V}$ & $1 K V$ & $Y \Lambda, Y$ & $\varepsilon I V$ & $r V, \varepsilon$ & ra1 & $r q, 7$ & $\varepsilon r 1$ & $r \leq$ \\
\hline rıo,. & $V Y, q$ & or. 1 & 0,9 & $\Lambda 4$ & $9, \cdot$ & $1 \pi 1$ & $r \Lambda, \varepsilon$ & हार & $r \wedge, r$ & ह11 & $r \Lambda, 0$ & $\leq 1 \leq$ & ro \\
\hline$\varepsilon 19, r$ & $V T, \Sigma$ & orry & $0, \varepsilon$ & $\mathrm{VA}$ & $\Lambda, 0$ & $1 K r$ & $r \Lambda, q$ & $\{r \mid$ & $r \wedge, 0$ & $\leqslant 10$ & $Y \Lambda, Y$ & $\leq 11$ & ry \\
\hline$\varepsilon \vee \wedge, r$ & $V \varepsilon, 1$ & orq1 & $\varepsilon, 0$ & 70 & $v, \tau$ & 11. & $r q, \wedge$ & $\varepsilon r \varepsilon$ & $r q, r$ & SYT & $r \wedge, q$ & $\varepsilon r$. & $r V$ \\
\hline$\varepsilon \wedge q, r$ & $V r, \Lambda$ & orra & $\varepsilon, \varepsilon$ & $7 \varepsilon$ & $\mathrm{v}, 0$ & 1.9 & $r q, 9$ & $\varepsilon r_{0}$ & $r, r$ & $\leqslant 0 \leqslant$ & $r v,$. & rar & $r \Lambda$ \\
\hline orr, 9 & $V \varepsilon, \cdot$ & orsl & $r, 9$ & ov & $\mathrm{v}, \cdot$ & $1 . r$ & $\Gamma \cdot, \varepsilon$ & $\varepsilon \leqslant Y$ & $r T, V$ & $\sum V V_{1}$ & Yצ, & TVA & rq \\
\hline$r q 4, \wedge$ & $V Y, O$ & orvA & $0, \wedge$ & 10 & $\Lambda, 9$ & 15. & $r \Lambda, 0$ & $\leqslant 1 \leqslant$ & $r \cdot, r$ & $\varepsilon r q$ & $r \uparrow, T$ & rAv & $r$. \\
\hline OYA,V & $V \leqslant, 0$ & $0 \leqslant r$. & $r, \wedge$ & 00 & 7,9 & $1 \ldots$ & $r \cdot, 0$ & $\varepsilon \leqslant \varepsilon$ & $r \cdot, v$ & $\leq \leqslant V$ & $Y \wedge, 1$ & $\varepsilon .9$ & $\mu$ \\
\hline$r ד V, V$ & $V Y, Y$ & oroo & $7, r$ & 91 & $9, r$ & $1{ }^{1}$ & $\mathrm{rA}_{\mathrm{\Lambda}, \mathrm{.}}$ & $\varepsilon \cdot 1$ & $r q, \mathrm{~V}$ & $\varepsilon r T$ & $Y \Psi, V$ & rM & rr \\
\hline or, r, & $V \varepsilon, V$ & $0 \leqslant T_{0}$ & $r, \wedge$ & 07 & 7,9 & 1.1 & $\Gamma \cdot, \varepsilon$ & $\varepsilon \leqslant r$ & $r q, r$ & $\varepsilon r V$ & $r q, \varepsilon$ & 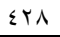 & r \\
\hline
\end{tabular}

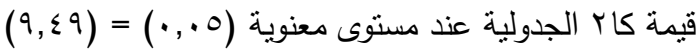

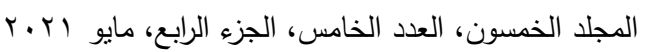

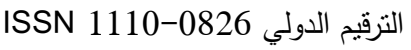




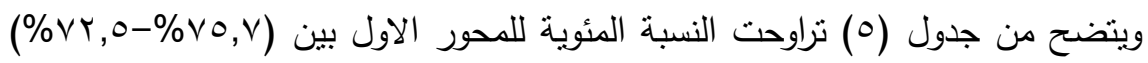

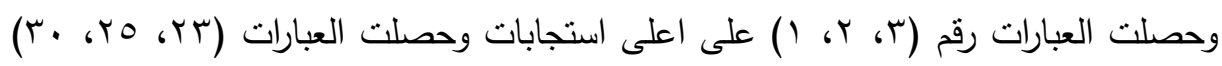
على ادنى استجابات وفيما يلى مناقثة العبارات.

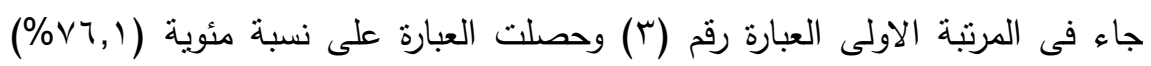

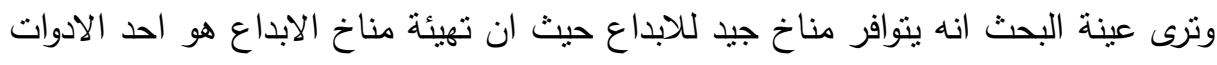
الرئيسية للنطوير ولابد من التجديد والابتكار فى وضع اليات التتفيذ للاستراتيجية لضمان

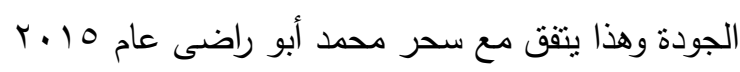

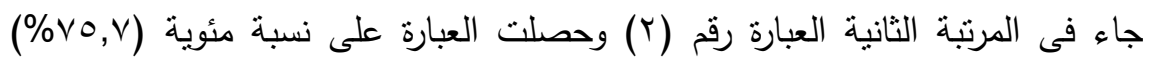
وترى عينة البحث ان الرؤية والرسالة الخاصة بالمدرسة لابد ان تتضمن التكنولوجيا الحديثة

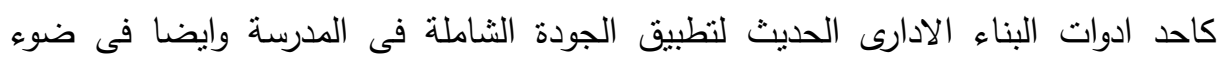
اللامركزية حتى لايتم اعاقة تتفيذ الروئية والرسالة والاهداف الاستراتيجية الخاصة بالمدرسة

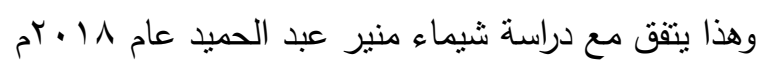

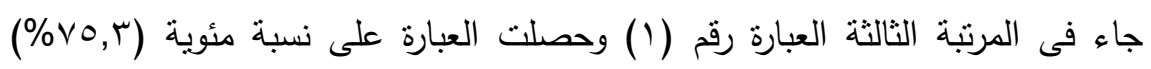
وترى عينة البحث ان وضع استراتيجية واضحة المعالم فى ضوء الاستراتيجية الوطنية للدولة

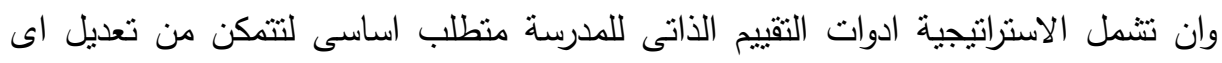

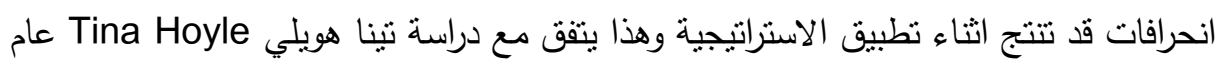
r...人 جاء فى المرتبة واحد وثلاثتن العبارة رقم (rr) وحصلت العبارة على نسبة مئوية (\%VY,1) الاساليب الحديثة التى يتم استخدامها فى طرق التدريس وذللك لتوجيه الطلاب للتفكير العلمى لتصى

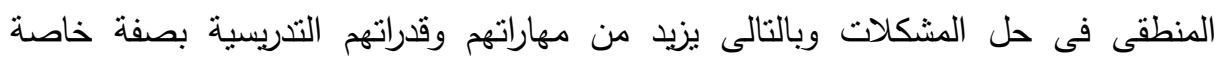

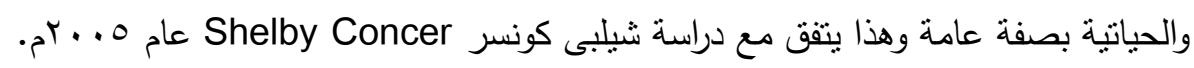


جاء فى المرنبة الثانية وثلاثون العبارة رقم (Y0) وحصلت العبارة على نسبة مئوية

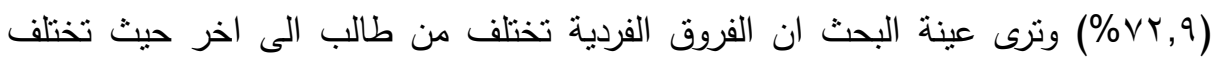

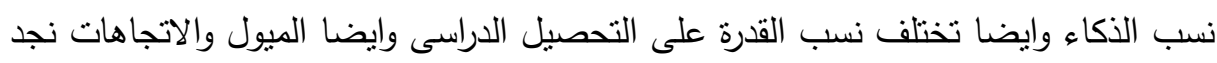
ان بعض الطلاب له ميول علمية واخرى ادبية وهكذا لذا لابد من الوضع فى الاعتبار التعامل

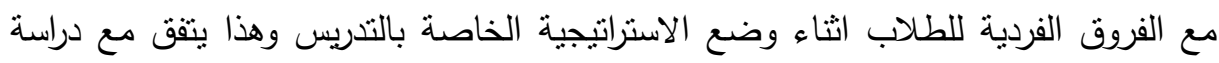

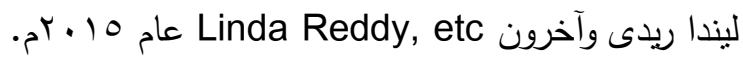
جاء فى المرنبة الثالثة وثلاثثن عبارة العبارة رقم (•r) وحصلت العبارة على نسبة مئوية

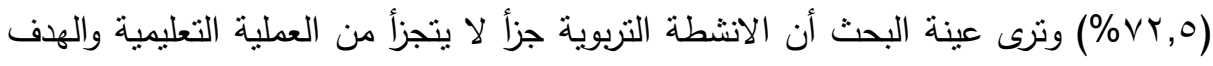
منها الاثراء الفكرى والمعرفى وربط العمليات التربوية بالانشطة من اجل زيادة منعة التعلم

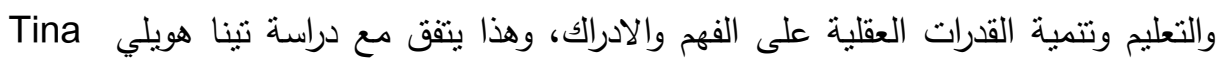
Hoyle 


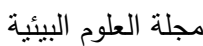

معهد الدراسات والبحوث البيئية - جامعة عين شمس البية

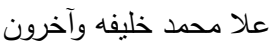

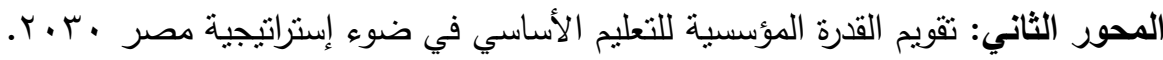
جدول (†): النكرارات والاهمية النسبية وكاץ لعبارات المحور الثاني (ن= 00ء ( )

\begin{tabular}{|c|c|c|c|c|c|c|c|c|c|c|c|c|c|}
\hline \multirow[t]{2}{*}{ SاY } & \multirow[t]{2}{*}{ النسبية } & \multirow{2}{*}{ اللرجات } & \multicolumn{2}{|c|}{ غيرة موافق } & \multicolumn{2}{|c|}{ غير موافق } & \multicolumn{2}{|c|}{ أوافق الى حـ } & \multicolumn{2}{|r|}{ اوفق } & \multicolumn{2}{|c|}{ أوافق بثدة } & \multirow[t]{2}{*}{ العبــارة } \\
\hline & & & $\%$ & ك & $\%$ & s & $\%$ & 5 & $\%$ & s & $\%$ & s & \\
\hline$r v v, q$ & $v_{1},$. & 0174 & $7, Y$ & 9. & $9, \wedge$ & $1 \leqslant r$ & $r \cdot, v$ & $\leqslant \leq 7$ & $r q, q$ & $\varepsilon \Gamma_{0}$ & $r r, 0$ & $r \leqslant r$ & 1 \\
\hline$\sum r V, A$ & $v_{1}, \varepsilon$ & 0197 & $0, \varepsilon$ & $\mathrm{Vq}$ & $9, \cdot$ & $1 \pi 1$ & $r r, q$ & $\varepsilon \vee \wedge$ & $Y \Lambda, 0$ & ¿1 & $r \varepsilon, r$ & ror & r \\
\hline $0 \vee 0,1$ & $v r, q$ & orvo & $r, r$ & $\leq 7$ & $7, \mathrm{~V}$ & 91 & $r \varepsilon, r$ & ¿१V & $r q, \varepsilon$ & $\sum \curlyvee \wedge$ & $r 4,0$ & rAT & r \\
\hline$\{r 0,0$ & V), T & or.A & $0, r$ & VV & $\wedge, 9$ & 149 & $r, q, q$ & $\varepsilon \vee \wedge$ & $r \Lambda, T$ & $\sum 17$ & $r \leqslant, \varepsilon$ & roo & $\varepsilon$ \\
\hline ovv, q & $V \varepsilon, \varepsilon$ & $0 \leqslant 1$. & $r,$. & $\varepsilon \varepsilon$ & 7,7 & 97 & rY,T & $\varepsilon V \varepsilon$ & $r 1,1$ & sor & $r \Psi, Y$ & 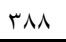 & 0 \\
\hline $0 \wedge V, \Lambda$ & vo,. & $0 \leqslant 00$ & $r,$. & $\varepsilon r$ & 7,0 & 90 & $r q, q$ & \&ro & r & 纟qr & 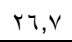 & rAq & 7 \\
\hline $0.9,1$ & $v r, 1$ & orit & $\varepsilon, 1$ & 09 & $v, 7$ & 111 & $r r, q$ & $\varepsilon \vee \wedge$ & $r q, \wedge$ & $\varepsilon r \varepsilon$ & $r_{0,7}$ & TVT & v \\
\hline or $\leqslant, 1$ & $V Y, q$ & or... & $\varepsilon, \cdot$ & 01 & $v, 7$ & 11. & $r \varepsilon, \varepsilon$ & $0 .$. & $r \Lambda, \varepsilon$ & हाr & ro,v & $r V \varepsilon$ & $\wedge$ \\
\hline orv, T & $V r, \varepsilon$ & ors. & $r, \wedge$ & 00 & $\mathrm{v}, \varepsilon$ & $1 . v$ & rr, q & $\varepsilon V A$ & $r \cdot, l$ & $\varepsilon$ ¿ & $r_{0,9}$ & rVv & 9 \\
\hline$\varepsilon r 0, V$ & $V Y, 1$ & Or $\leqslant 0$ & $0, \varepsilon$ & $V \lambda$ & $\Lambda, 9$ & $1 \pi$. & $r q, 9$ & $\varepsilon r_{0}$ & $r 1,0$ & $\{01$ & $r \varepsilon, r$ & ros & 1. \\
\hline$\leqslant \leqslant 1,9$ & $\forall Y, \varepsilon$ & orto & 0,1 & $V \varepsilon$ & $\Lambda, \nu$ & $1 K 4$ & $r \cdot, r$ & $\varepsilon r q$ & $r_{1,0}$ & $\{01$ & $r \varepsilon, T$ & rod & 11 \\
\hline 纟or, 9 & $V Y, O$ & orri & $\varepsilon, 9$ & VI & $\Lambda, 0$ & אז & $r,$. & $\leq 01$ & $r \cdot, q$ & $\leq \leqslant 9$ & $r \leqslant, A$ & (וד & Ir \\
\hline$\leq \leqslant 0,0$ & $V Y, r$ & ortl & $0,$. & $V T$ & $\Lambda, 7$ & iro & $r \cdot, 9$ & $\leq \leqslant 9$ & $r \cdot, 9$ & $\leq \leqslant 9$ & $r \leqslant, V$ & roq & $1 T$ \\
\hline$\{r), \cdot$ & 51,9 & OrTs & $0, \varepsilon$ & 19 & $9, \cdot$ & $1 \pi 1$ & $r \cdot, r$ & $\leq \varepsilon$. & $r 1,1$ & Eor & $r \leqslant, r$ & ror & $1 \leq$ \\
\hline$\leqslant v_{0}, q$ & $V r,$. & or. 1 & $\varepsilon, 0$ & 77 & $\Lambda, 1$ & 111 & $r \cdot, 0$ & $\varepsilon \leqslant \varepsilon$ & $r 1, v$ & $\{71$ & ro,r & r47 & 10 \\
\hline$\sum \wedge १, \wedge$ & $v r, r$ & orrg & $\varepsilon, r$ & Tr & $v, q$ & 110 & $r \cdot, r$ & $\varepsilon \leqslant 1$ & $r, 1$ & $\sum 7 V$ & $r_{0, \varepsilon}$ & r4q & 17 \\
\hline$\leq v 1,7$ & 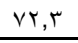 & OYTY & $\varepsilon, V$ & 71 & $\Lambda, r$ & Ir. & $r r, q$ & $\leqslant \vee \wedge$ & $r q, r$ & Ero & $r_{0, \text {, }}$ & TIE & IV \\
\hline 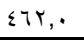 & $V Y, Y$ & oros & $\varepsilon, \Lambda$ & V. & $\Lambda, \varepsilon$ & IKY & rT, T & $\varepsilon V \leqslant$ & $r q, r$ & $\sum r V$ & $r \leqslant, 9$ & rit & 11 \\
\hline$\{\wedge \wedge, 9$ & $V r, 1$ & Or $\leqslant$ r & $\varepsilon, 7$ & TV & $\Lambda, r$ & 119 & $r \leqslant, T$ & $0 . r$ & $r V, T$ & $\leq .1$ & $r_{0,1}$ & ryo & 19 \\
\hline orr,o & $\mathrm{Vr}, 1$ & oriv & $\Gamma, \Lambda$ & 00 & $\mathrm{~V}, \varepsilon$ & $1 . v$ & $r \varepsilon, \varepsilon$ & 0.1 & $r \Lambda, 0$ & $\leqslant 10$ & ro, 9 & rVv & $r$. \\
\hline $0 . v, \varepsilon$ & $V r, r$ & OrTV & $\varepsilon, 1$ & 09 & $v, \tau$ & 111 & $r r, 1$ & $\sum T V$ & $r \cdot, \tau$ & $\leqslant \leqslant 0$ & Y0,T & rVT & r) \\
\hline$\varepsilon \wedge \cdot, r$ & $Y Y, 1$ & Or $\leq \leqslant$ & $\varepsilon, \mathrm{V}$ & 71 & $\Lambda, Y$ & ir. & $r \leqslant, 1$ & $\sum 97$ & $Y \Lambda,$. & $\varepsilon \cdot V$ & $r_{0},$. & TIE & $r r$ \\
\hline$\leq \leqslant १, \cdot$ & $\vee 1, \wedge$ & OrYY & 0,1 & $V \varepsilon$ & $\Lambda, \vee$ & $1 T_{4}$ & $r r, 1$ & \&AY & $r \wedge, 0$ & $\leqslant 10$ & $r \leq, T$ & ros & r \\
\hline$\leq \leqslant 9, \varepsilon$ & VY,. & OrTV & $0,$. & $V T$ & $\Lambda, \uparrow$ & Iro & Tr,O & $\varepsilon V T$ & $r q, r$ & sro & $r \leq, Y$ & roq & $r \varepsilon$ \\
\hline $09 \vee, 1$ & $V \varepsilon, 9$ & $0 \leqslant \leqslant A$ & $Y, V$ & $\varepsilon$. & $7, r$ & 94 & 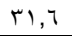 & $\leq 7$. & $T Y, \varepsilon$ & 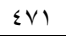 & $r q, 9$ & rar & ro \\
\hline
\end{tabular}

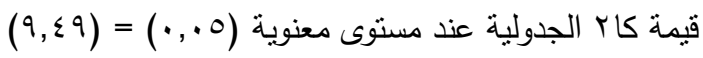




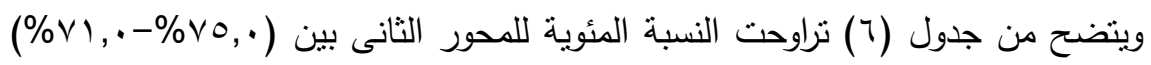

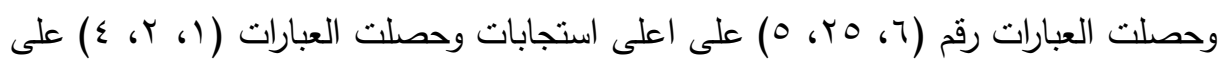
ادنى استجابات وفيما يلى مناقثنة العبارات.

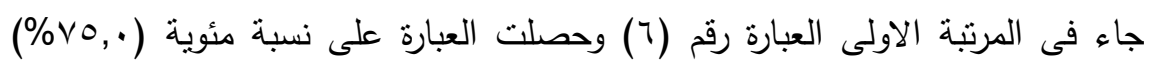
وترى عينة البحث ان محتوى المناهج لابد من تقييمها بصفة دورية والتعرف على الآليات الملائمة للتطبيق والمؤشرات السليمة لعميات التقويم كما يجب تعزيزها بالحقائب المدرسية وفقا

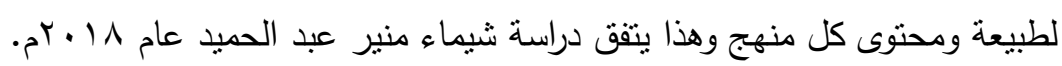

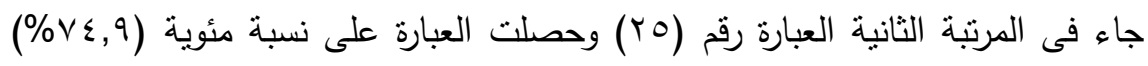
وترى عينة البحث ان تحليل سوق العمل يجب ان يتم قبل وضع آليات المخرجات المطلوبة

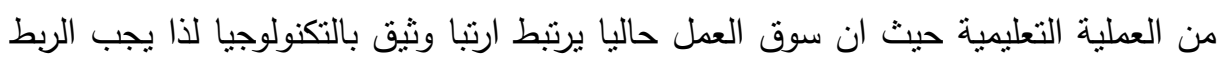

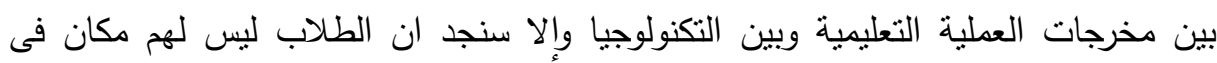

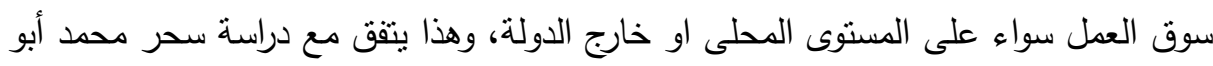

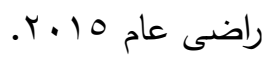

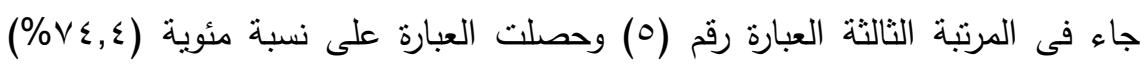
وترى عينة البحث ان الانشطة اللا صفية تعد عامل جذب واهتمام من قبل الطلاب اكثر منها

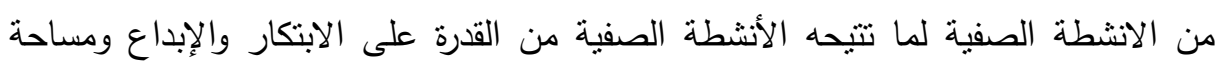

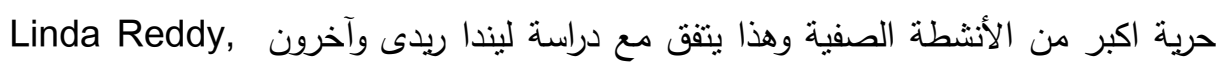
etc جاء في المرتبة الثالثة وعشرون العبارة رقم (1) وحصلت العبارة على نسبة مئوية

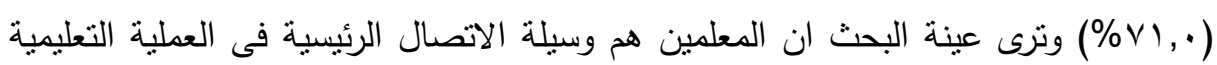

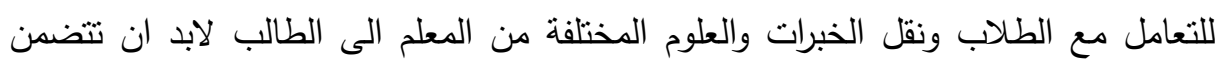

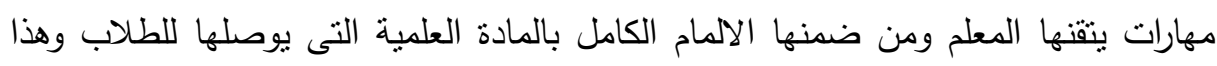

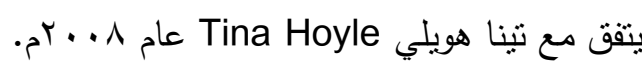


جاء فى المرنبة الرابعة وعشرون العبارة رقم (Y) وحصلت العبارة على نسبة مئوية (\%VY, ( )

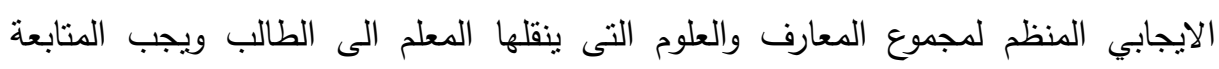
الدورية لسجلات تحضير الدروس للمعلمين واطلاعهم على كل ما هو جديد ومستحدث فى التخطيط للارس حيث ان التخطيط الجيد يضمن التقيذ الجيد وهذا يتفق مع دراسة شيماء منير

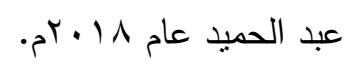
جاء فى المرتبة الخامسة وعشرون العبارة رقم (ع) وحصلت العبارة على نسبة مئوية

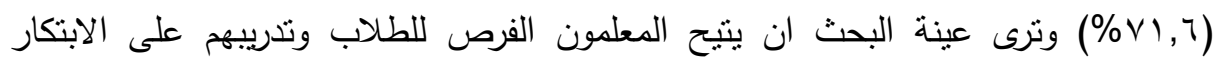

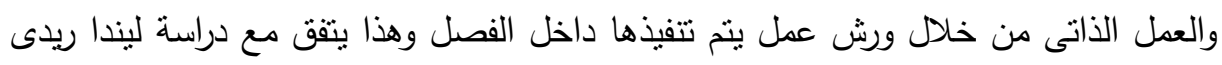

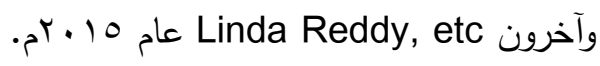

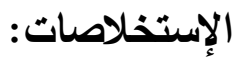
فى ضوء نتائج البحث ومناقتنتها توصل الباحثون الى الاستخلاصات الاتية: استخلاصات خاصة بالمحور الاول: الفاعلية التعليمية:

- تؤثر الفاعلية على عمل واداء المؤسسة التعليمية وهى تعبر ايضا عن مدى التماء التهاء العاملين

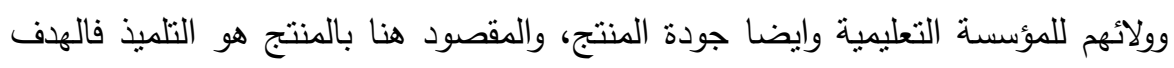
من زيادة الفاعلية او قياسها هو التعرف على دورها فى جودة المنتج وايضا رضا رضا اولياء الامور وايضا الأداء الاستراتيجي للمؤسسة التعليمية ككل.

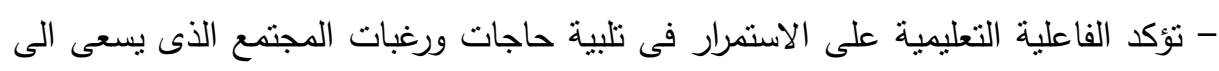

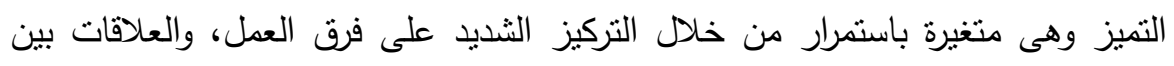
العاملين فى المؤسسة التعليمية لكى يؤمن فرص فئنسرة عديدة للتفاعل الاجتماعي والتوطيد

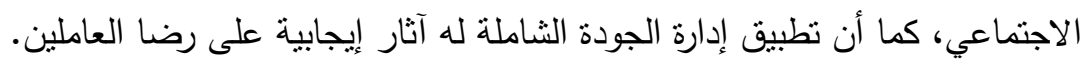


- يسهح وضع الاستراتيجيات فى رفع اداء انجاز التلاميذ فى إعداد القوة البشرية التي تحتاجها

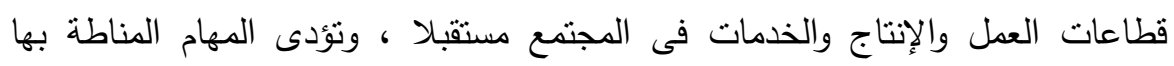
بكفاءة عالية.

- الاهتمام بإنجاز التلاميذ يتطلب أن تتوفر لها الموارد والإمكانيات المادية والبشرية اللازمة

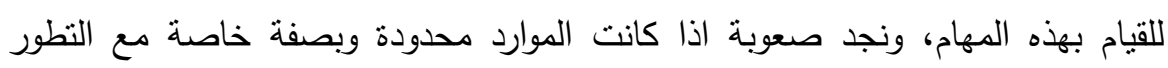
التقنى فائق السرعة الذى أحدث فجوة كبيرة بين النظم التعليمية، وبخاصة مع التهدة الانخفاض المستمر في الموارد المالية والمادية الممنوحة للمؤسسات التعليمية. استخلاصات خاصة بالمحور الثانى: القدرة المؤسسية - يتطلب تحقيق آليات الجودة فى المؤسسات التعليمية تصميم وتتفيذ نظام يتضمن سياسات وإجراءات للتأكد من الوفاء بمنطلبات الجودة، ليس فقط على نطاق ومراحل عملية إنتاج

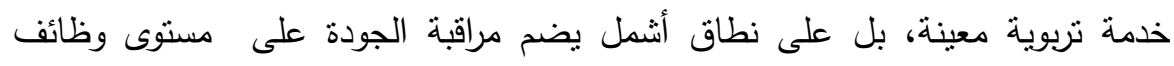

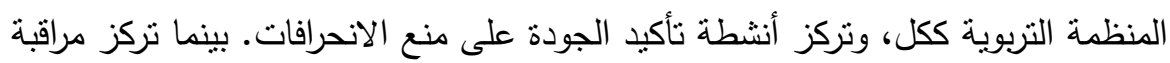
الجودة على كثفها أو اكتثافها بعد حدوثها. - تضع المدرسة فى ضوء ادارة الجودة الثناملة خطة للتحسين المستمر في ضوء نتأئج التقييم

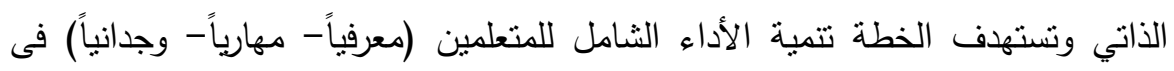

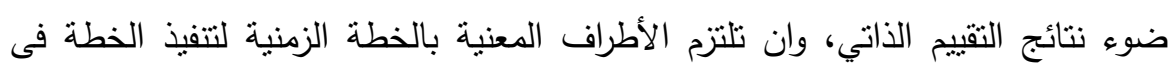

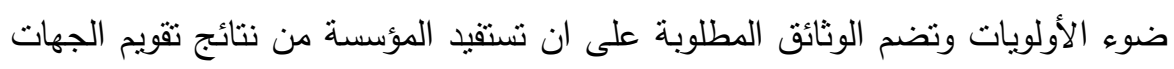

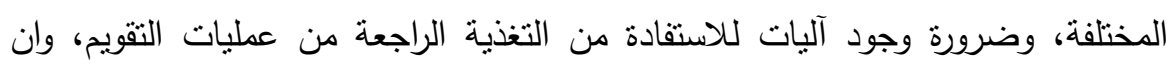
تتوافر تقارير موثقة للتقييمات من الخارج؛ للتأكد من مدى مطابقة أداءاتها لمعايير الجودة.

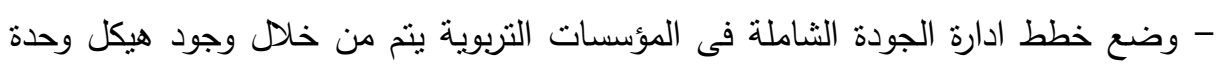
التدريب والتقويم بالمدرسة على ان تتبع وحدة ضمان الجودة، ويكون جزء من عملها توكيد

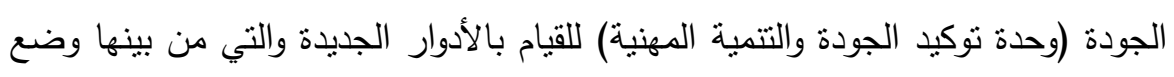




$$
\begin{aligned}
& \text { مجلة العلوم البيئية } \\
& \text { معهد الدراسات والبحوث البيئية - جامعة عين شمس لئه } \\
& \text { علا محمد خليفه وآخرون }
\end{aligned}
$$

تقرير عن الحالة التعليمية بالمدرسة، وربط عملية الاعتماد الأكاديمي بالتقويم، والتقويم والمتابعة، وإدارة التدريب، ومؤشرات الأداء، والتتسيق مع المدارس الأخرى.

\section{اللزوكياهي}

وفى ضوء مناقثة الباحثون وتحليلهم لعبارات ومحاور البحث وفى ضوء النتائج التى تم

$$
\text { التوصل إليها اقترح الباحثون : }
$$

\section{توصيات مرتبطة بالمحور الاول: الفاعلية التعليمية}

1- إنقوم المدرسة بإعداد خطة استراتيجية من خلال:

$$
\text { • إجراء تقييم ذاتى للمؤسسة. }
$$

• مشاركة جميع العاملين بالمدرسة في إعداد التقييم الذاتى.

• • مشاركة الأطراف المجتمعية ذات العلاقة فى التقييم.

r- استخدام استراتيجيات التدريس الحديثة التى تلبى احتياجات التلاميذ من خلال:

• ت تدريب المعلمون على استراتيجيات التدريس الحديثة .

• ت توفير التقنيات اللازمة لتطبيق استراتيجيات التدريس الحديثة.

ب- تلتزم الددرسة بالحفاظ على الجودة وتحسينها من خلال :

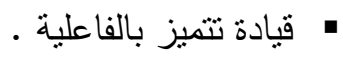
• مشاركة نشطة من جميع العاملين فى المدرسة.

ع- يتم دمج عمليات الثقويم وتخطيط عمليات تحسين الجودة فى العمليات الادارية المعتادة

$$
\text { وتصبح جزء منها. }
$$

0- وجود مركز للجودة ضمن هيكل الادارة المركزية للمدرسة ويتولى المهام الاتية:

$$
\text { • تتفيذ ملاحظات فريق التحسين الخارجي بدقة. }
$$

• الاهتمام بالموارد البشرية وجودتها لضمان جودة المخرجات من العملية التعليمية.

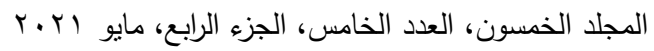

$$
\begin{aligned}
& \text { الترقيم الدولي 0826- النيان }
\end{aligned}
$$


• وجود آليات تستخدم في تقييم أداء التنلاميذ مناسبة لأنماط التعلم المطلوبة 7- سعى ادارة المدرسة الى تهيئة البيئة التعليمية المحفزة للتعليم والتعلم من خلال: • توفير الامكانات المادية المحفزة للتعليم والتعلم • توفير الامكانات البشرية المؤهلة، وتوفير التقينات الحديثة. • الاهتمام بالموارد البشرية وجودتها لضمان جودة المخرجات من العملية التعليمية. V- تحسين اداء التلاميذ من خلال • اكساب المهارات الحسابية كههارة الضرب وحل المسائل الحسابية، والقراءة الجهرية، والتعبير الكتابي والقواعد الإملائية والنحوية. • اتقان مهارات تقنية المعلومات، ومراعاة الفروق الفردية التلاميذ. • توبع الأنشطة الصفية واللاصفية. • تفعيل أنشطة وفعاليات تفعل التكامل المعرفي للتلاميذ. توجيه الأنشطة التعليمية والتربوية لتتمية جميع جوانب شخصية التلميذ. توصيات مرتبطة بالمحور الثانى: القدرة المؤسسية: 1- تلتزم ادارة المدرسة بتطوير القدرة المؤسسية من خلال قيادة فاعلة ومشاركة نشطة من جميع العاملين فى المدرسة من خلال: اثراء الأنشطة اللاصفية لخبرات واهتمامات التنلاميذ. مراعاة ظروف المتغيرات التعليمية من خلال تحقيق المدرسة للمعايير بصفة مستمرة ومنتظمة.

تشجيع الابتكار والإبداع على كل المستويات في المدرسة ويكون ذلك مصحوباً بسياسات

$$
\text { وإرشادات واضحة. }
$$

تتاول التقويُُ المدخلاتِ، والعمليات، والنواتج (المخرجات) مع التركيز على جودة النواتج.

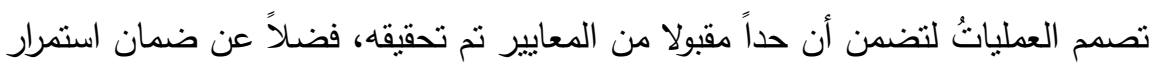

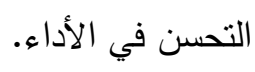


" اختيار عدد محدود من مؤشرات الأداء الأساسية التي تقدم أدلة وبراهين قادرة على التى القياس بشكل موضوعي على جودة الأداء فى المدرسة.

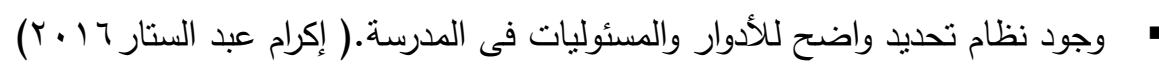

\section{المرالئar}

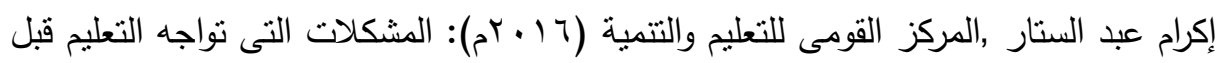

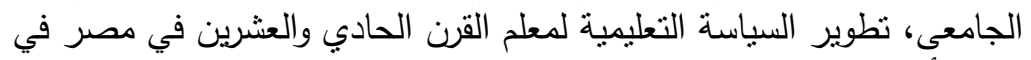

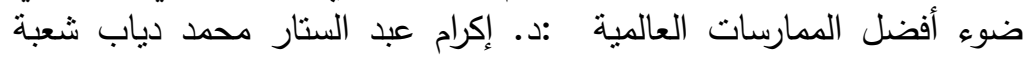
بحوث المعلومات التربوية، القاهرة.

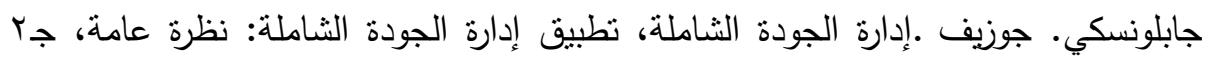

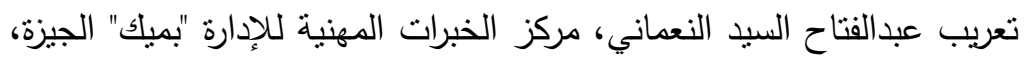

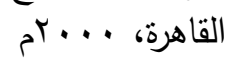

محمد عبد الوهاب العزاوي :الجودة الثاملة في التعليم العالي الندوة الأولى: إدارة الجودة

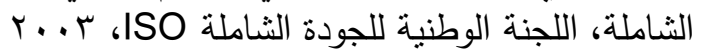

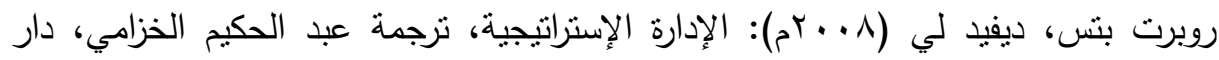

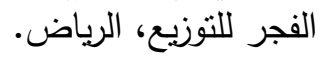

سحر محمد أبو راضى (10 • بام): تفعيل ثقافة المساءلة التزبوية لدى معلىي التعليم الثانوي

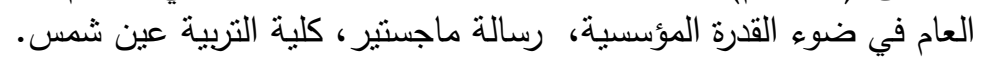

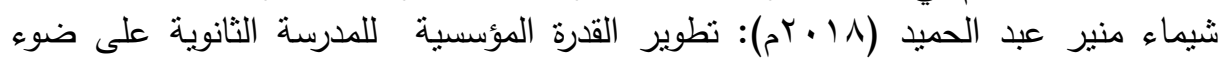

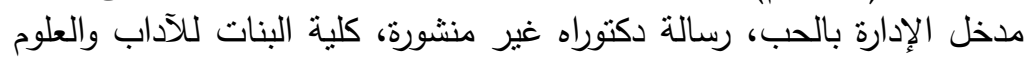
والتربية قسم اصول التربية، القاهرة.

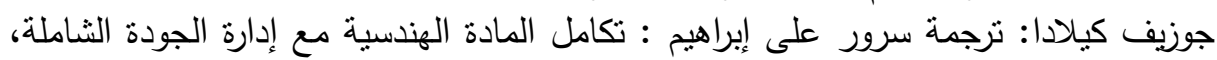

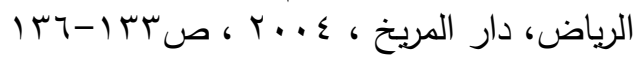

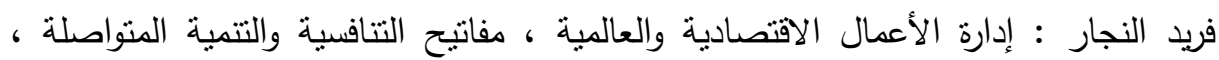
مؤسسة شباب الجامعة ، الإسكندرية ، 1991 ، ، صلح الإل

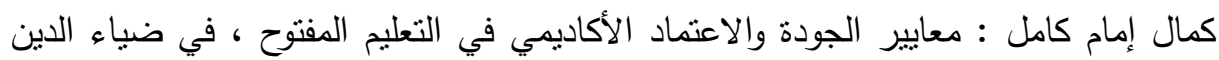

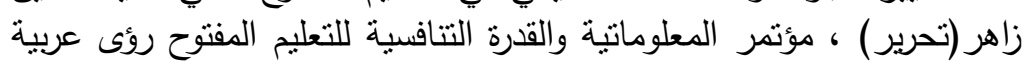

$$
\begin{aligned}
& \text { المجلد الخمسون، العدد الخامس، الجزء الرابع، مايو الr.r. }
\end{aligned}
$$

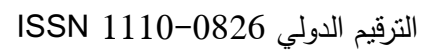




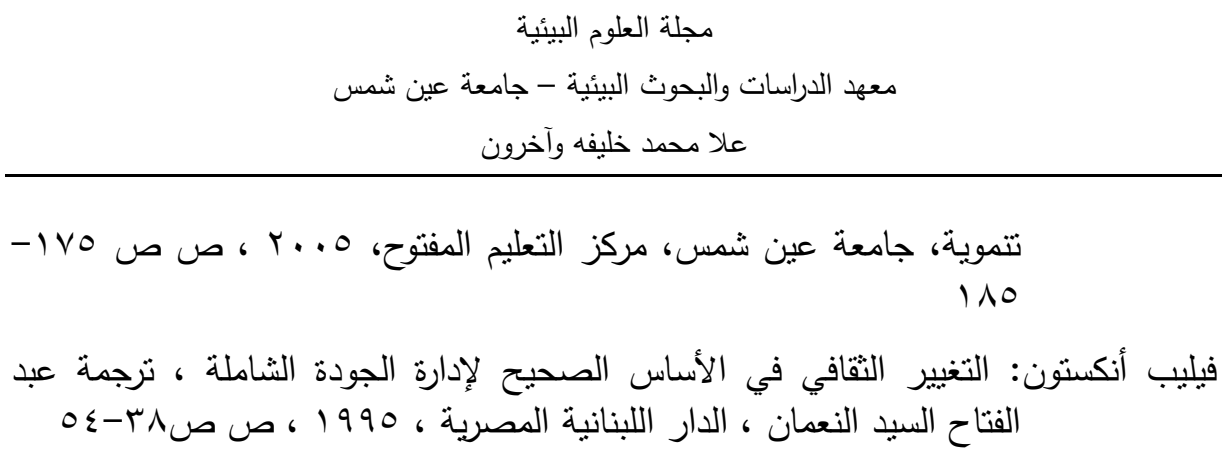

Alton L. Taylor \& Carol M. Pfeiffer (2000) "Strategic Changein College and University", the Journal of Higher Education, Columbus, Vol. 71, Issue 4, Jul/Agu

(Crosby, Philip1996)Quality is still free: making quality certain in uncertain times. New York/London, pp 145 ,McGraw-Hill, 1996

Dale, B.G. and Plunkett, J.J. (1995) Quality Costing. 2nd Edition, Chapman and Hall, London

Frederick. F and Teal Thomas, the loyalty effect: the hidden force behind growth, profits and lasting value, Boston: Mass, Harvard business School press, 1996, p: 12-13.

Judeh, Mahfuz. (2012). إدارة الجودة الثشاملة: مفاهيم وتطبيقات

JURAN, J. M., \&Gryna, Frank M, -Juran's Quality Control Handbook, 4th ed., New York: McGraw-hill 1998

Linda A.Reddy,Ryan J.Kettler\& Alexander kurz (2015) : Institutional Capacity and High School Marketing, Journal of Educational and Psychological Consultation, Vol (25), issue(2-3),

Porter Michael,; l'Avantage concurrentiel, Comment devancer ses concurrents et maintenir son avancé, DUNOD édition, Paris, 2016

Shelby Conser (2009) : Building Organizational Capacity Through Trust, Educational Administration Quarterly, Vol.45, No.2,

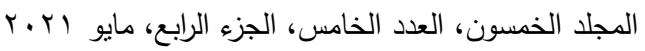

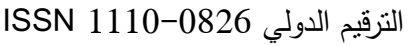




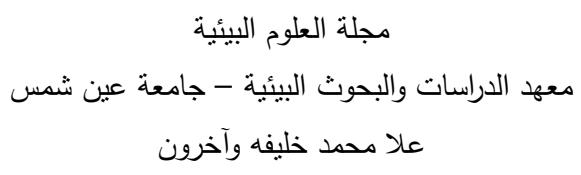

Tina B. Hoyle (2008): Building Capacity For The Continuous Improvements Of Health-Promoting Schools, Journal of School Health, Vol.78, No.1,

UNESCO (2003) Institute for Education (,NURTURING THE TREASURE Vision and Strategy 2002 - 2007, Hamburg, Germany, pp14

UNESCO, (2004) EFA Global Monitoring Report 2005, THE QUALITY IMPERATIVE Education for All, the United Nations Educational Scientific and Cultural Organization, pp 5

UNESCO:2015, The Human Capital Report, Employment, Skills and Human Capital Global Challenge, 2015

Internet

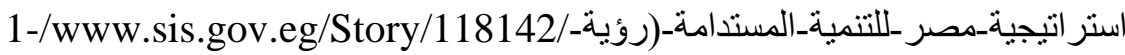
$r \cdot r \cdot-r)$ (مصر)?

2-https://sites.google.com/site/ahmad7ghtq/gu/almjmwtealthanyte/Ishikawa 


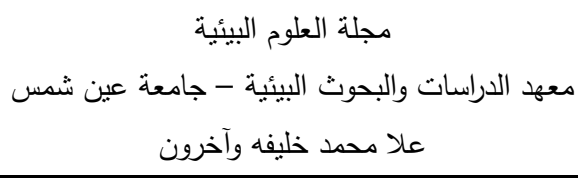

\title{
MECHANISMS FOR EVALUATING THE TOTAL QUALITY MANAGEMENT OF THE BASIC EDUCATION STAGE IN LIGHT OF EGYPT'S 2030 STRATEGY
}

\author{
Ola M. Khalifa ${ }^{(1)}$; Mamdouh A. Rifai(2) Suzan M. Al Mahdi( ${ }^{(3)}$; \\ and Magda M. Gabriel ${ }^{(2)}$ \\ 1) Port Said School Zamalek 2) Faculty of Commerce, Ain Shams \\ University 3) Basic of Education Girls College, Ain Shams University
}

\begin{abstract}
Egypt is now developing and modernizing education via the administrations which cope with the age requirements. Those emphasize quality and competitive ability. Nevertheless, basic education in Egypt is confronted by various problems to reach assessment mechanics in addition to lack of formulating a paramount vision of syllabuses and school activities in order to reach common concepts which link workers, students and the local society. Besides, Students have no say about the syllabuses they study or the methods used to teach those syllabuses. Consequently, this weakens the application of quality pointers in schools. In addition, there are reasons related to the educational organizations themselves such as relying on traditional teaching methods, lack of professional development, the wide gap between the training programs and real needs of development, the weakness of school administrations to achieve reformation of quality and finally the lack of understanding and integration in internal functional roles.
\end{abstract}

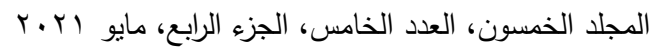

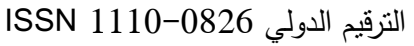




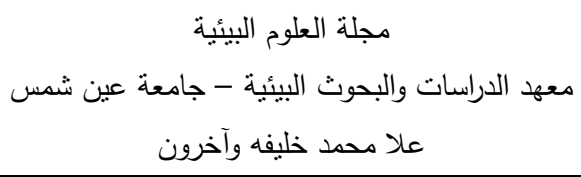

This research aims through seeking answers to the research questions concerning formulating assessment mechanics to the basic education total quality administration in the light of Egypt 2030 Strategy through: Assessing the basic stage effectiveness in general in the light of Egypt Strategy 2030. And Assessing the basic stage quality and accountability mechanics in the light of Egypt Strategy 2030. The importance of this research is that it is hoped to lend a helping hand to policy makers and decision takers in educational administrations to recognize the downsides of traditional school administrations, and how far workers are in need of such a kind of administration in order to be able to do their best. It is also hoped that the study presents a suggested concept of the mechanics to assess total quality. That concept is to cope with the world trends and the current efforts to reform education and which focus on achieving quality in educational organizations. Among the most important recommendations are the necessity of using modern teaching strategies which satisfy the students' needs through training teachers to the recent strategies and providing the required technological methods to apply those strategies in order to better the internal competence through modifying the quality and quantity of inputs so that they can be used in the best means ever. he study was characterized by objective, human, place and time limitations. By objective limitations, the study was restricted to assessing effectiveness in general and assessing the mechanisms of quality and accountability needed to basic stages of education. Human limitations mean that the field study comprised a random sample of zone directors, heads of sectors, boards of trustee members in addition to school directors. Place limitations refer to some schools in Cairo, Behira, and The New Valley Governorates. Finally, time limitations signify that the field study was applied in the first semester of the school year 2019-2020

Keywords: Total Quality Management Assessment Mechanisms Basic Education

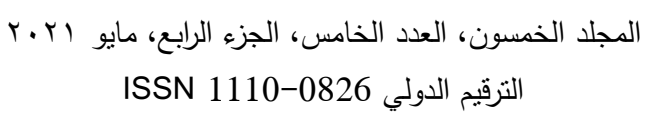

